\title{
Panel error correction testing with global stochastic trends
}

Citation for published version (APA):

Gengenbach, C., Urbain, J. R. Y. J., \& Westerlund, J. (2008). Panel error correction testing with global stochastic trends. METEOR, Maastricht University School of Business and Economics. METEOR Research Memorandum No. 051 https://doi.org/10.26481/umamet.2008051

Document status and date:

Published: 01/01/2008

DOI:

10.26481/umamet.2008051

Document Version:

Publisher's PDF, also known as Version of record

\section{Please check the document version of this publication:}

- A submitted manuscript is the version of the article upon submission and before peer-review. There can be important differences between the submitted version and the official published version of record.

People interested in the research are advised to contact the author for the final version of the publication, or visit the DOI to the publisher's website.

- The final author version and the galley proof are versions of the publication after peer review.

- The final published version features the final layout of the paper including the volume, issue and page numbers.

Link to publication

\footnotetext{
General rights rights.

- You may freely distribute the URL identifying the publication in the public portal. please follow below link for the End User Agreement:

www.umlib.nl/taverne-license

Take down policy

If you believe that this document breaches copyright please contact us at:

repository@maastrichtuniversity.nl

providing details and we will investigate your claim.
}

Copyright and moral rights for the publications made accessible in the public portal are retained by the authors and/or other copyright owners and it is a condition of accessing publications that users recognise and abide by the legal requirements associated with these

- Users may download and print one copy of any publication from the public portal for the purpose of private study or research.

- You may not further distribute the material or use it for any profit-making activity or commercial gain

If the publication is distributed under the terms of Article $25 \mathrm{fa}$ of the Dutch Copyright Act, indicated by the "Taverne" license above, 
Christian Gengenbach, Jean-Pierre Urbain, Joakim Westerlund

Panel Error Correction Testing with Global Stochastic Trends

$\mathrm{RM} / 08 / 051$

JEL code: C12, C33

\section{METE@R}

Maastricht research school of Economics of TEchnology and ORganizations

Universiteit Maastricht

Faculty of Economics and Business Administration P.O. Box 616

NL - 6200 MD Maastricht

phone : ++31433883830

fax : ++31433884873 


\title{
Panel Error Correction Testing with Global Stochastic Trends*
}

\author{
Christian Gengenbach \\ Universiteit Maastricht \\ The Netherlands
}

\author{
Jean-Pierre Urbain \\ Universiteit Maastricht \\ The Netherlands
}

\author{
Joakim Westerlund ${ }^{\dagger}$ \\ Lund University \\ Sweden
}

December 15, 2008

\begin{abstract}
This paper considers a cointegrated panel data model with common factors. Starting from the triangular representation of the model as used by Bai et al. (2008) a Granger type representation theorem is derived. The conditional error correction representation is obtained, which is used as a basis for developing two new tests for the null hypothesis of no error correction. The asymptotic distributions of the tests are shown to be free of nuisance parameters, depending only on the number of non-stationary variables. However, the tests are not cross-sectionally independent, which makes pooling difficult. Nevertheless, the averages of the tests converge in distribution. This makes pooling possible in spite of the cross-sectional dependence. We investigate the finite sample performance of the proposed tests in a Monte Carlo experiment and compare them to the tests proposed by Westerlund (2007). We also present two empirical applications of the new tests.
\end{abstract}

Keywords: Panel cointegration, common factors.

\footnotetext{
*Previous versions of this paper were presented at the 2008 conference on Factor Structures for Panel and Multivariate Time Series Data in Maastricht and at a seminar at Lund University. The author would like to thank conference and seminar participants, and in particular David Edgerton, Maria Holmlund, Barry Jones and Franz Palm for many valuable comments and suggestions. Westerlund would also like to thank the Maastricht Research School of Economics of Technology and Organizations for its hospitality during a visit at the Department of Quantitative Economics at Universiteit Maastricht, where this work was initiated. Financial support from the Jan Wallander and Tom Hedelius Foundation under research grant number W2006-0068:1 is gratefully acknowledged.

${ }^{\dagger}$ Corresponding author: Department of Economics, Lund University, P. O. Box 7082, SE-220 07 Lund, Sweden. Telephone: +46 46222 8670, Fax: +46 46222 4118, E-mail address: joakim.westerlund@nek.lu.se.
} 


\section{Introduction}

Consider two non-stationary panel data variables $X_{i, t}$ and $Y_{i, t}$, where $i=1, \ldots, N$ and $t=$ $1, \ldots, T$ indexes the cross-sectional and time series dimensions, respectively. The analysis of such variables has been a growing field of econometric research in recent years. See for example Breitung and Pesaran (2008) for an overview. In particular, in many economic applications it is an important question whether $X_{i, t}$ and $Y_{i, t}$ are cointegrated, that is whether there exists a meaningful long-run relationship between them, or whether the relationship is spurious.

Kao (1999) and Pedroni $(1999,2004)$ were among the first to propose residual-based tests for the null hypothesis of no cointegration in cross-sectionally independent panels. But crosssectional independence is a restrictive assumption that is unlikely to be met in practice, in which case the properties of this kind of tests become suspect. In fact, in a recent paper, Gengenbach et al. (2006) show that the presence of cross-section dependence in the form of non-stationary common factors can actually cause the residual-based tests of Kao (1999) and Pedroni (2004) to become divergent. As a response to this, they propose to estimate separately the common and idiosyncratic components of $X_{i, t}$ and $Y_{i, t}$ using the principal components method of Bai and $\mathrm{Ng}$ (2004), and then to test for cointegration in the resulting component estimates.

Banerjee and Carrion-i-Silvestre (2006) propose a similar test but instead of applying the Bai and $\mathrm{Ng}$ (2004) approach to $X_{i, t}$ and $Y_{i, t}$ directly, they apply it to the residuals of a first-stage regression of $Y_{i, t}$ onto $X_{i, t}$. Cointegration requires that both the common and idiosyncratic components of the residuals are stationary. The tests of Bai and Carrion-iSilvestre (2007), Westerlund (2007) and Westerlund and Edgerton (2008) are basically the same in the sense that they are also based on applying the Bai and $\mathrm{Ng}$ (2004) approach to the residuals of a first-stage regression.

However, although very popular, this testing approach has at least two major drawbacks. One lies with the use of residual rather than structural dynamics, which makes it subject to the common factor critique of Kremers et al. (1992), that may lead to tests with low power. The second drawback is that the testing must be carried out in steps, with the estimation error from one step being imported into subsequent steps, and it is not fully clear what effect this has on the final test, see Westerlund and Larsson (2008).

By contrast to the test proposed by Pedroni for example, the tests of Westerlund (2007) are not based on residuals but rather on the significance of the error correction term in a conditional panel error correction model (ECM), and therefore do not impose any common

factor restriction. However, the tests are derived under cross-sectional independence, and the use of the bootstrap in case of violations does not fit well with the otherwise parametric flavor of the tests. Another drawback is that the bootstrap used is not equipped to handle the case with non-stationary common factors. 
The current paper can be seen as an attempt to overcome the drawbacks of both these approaches. We begin by developing alternative representations of a cointegrated panel that allows for the possibility of non-stationary common factors. In particular, starting from the triangular representation of the system used by for example Bai et al. (2008), we derive a Granger type representation theorem that is similar to the one obtained by Cappuccio and Lubian (1996) in the case of a single time series.

The Granger representation theorem provides not only moving average (MA) and autoregressive moving average (ARMA) representations of the system, but also the conditional ECM representation, which we use as a basis for developing tests for the null hypothesis of no error correction. In particular, paralleling the development of the time series literature in this field, as pioneered by Banerjee et al. (1998) and Boswijk (1994), we consider both a $t$-ratio type test, as well as a Wald type test. Besides eliminating the need for a common factor assumption and a stepwise testing procedure, as shown by Pesavento (2004), these tests are not only more powerful than most residual-based tests around, but are also not worse in terms of size distortions.

It is shown that at the level of the individual unit the asymptotic distribution of the Wald tests is free of nuisance parameters and only depends on the number of non-stationary variables in the system. For the $t$-ratio an appropriate correction has to be employed to remove the nuisance parameter dependence from the limiting distribution. Nevertheless, because of the common factors, the individual tests are not independent, which of course makes pooling, or cross-sectional averaging, difficult, as it invalidates the use of the conventional limit theory. However, although not analytically tractable, the average still converges to a random variable with a distribution that can be easily simulated, which makes pooling possible in spite of the dependence. We begin by considering the case when the common factors are known, and then we show how the results extend to the case when the factors are approximated by means of cross-sectional averages of the observed data, as suggested by Pesaran (2007).

The rest of this paper is organized as follows. Section 2 presents the model of interest and our version of the Granger representation theorem. Sections 3 and 4 then present the error correction tests and their asymptotic properties, which are verified using both simulated and real data in Sections 5 and 6, respectively. Section 7 concludes.

A word on notation. The symbols $\stackrel{w}{\longrightarrow}$ and $\stackrel{p}{\longrightarrow}$ will be used to signify weak convergence and convergence in probability, respectively. As usual, $X_{T}=O_{p}\left(T^{r}\right)$ will be used to signify that $X_{T}$ is at most order $T^{r}$ in probability, while $X_{T}=o_{p}\left(T^{r}\right)$ will be used in case $X_{T}$ is of smaller order in probability than $T^{r}$. In the case of a double indexed sequence $X_{N, T}$, $N, T \rightarrow \infty$ will be used to signify that the limit has been taken while passing both indices to infinity jointly. For a square matrix $A, r k(A), \operatorname{adj}(A)$ and $\|A\|$ will denote its rank, adjoint and Euclidian norm, respectively. For simplicity, the Brownian motion $B(s)$ defined on the 
interval $s \in[0,1]$ will be written $B$, with the measure of integration omitted. We write the integral $\int_{0}^{1} B(s) d s$ as $\int B$ and $\int_{0}^{1} B(s) d B(s)^{\prime}$ as $\int B d B^{\prime}$. Finally, $\lfloor x\rfloor$ will be used to denote the integer part of $x$.

\section{Model representation}

In this section we discuss the model under consideration, and some alternative representations thereof. We start from the triangular representation for a single unit $i$, which is the same as the one used by Bai et al. (2008). However, these authors focus on how to conduct inference if the variables are in fact long-run related, and do not consider the problem of how to test for cointegration. Moreover, the triangular representation is taken as given, and there is no consideration of other alternatives. Thus, the results reported herein can in many ways be seen as complementary to those reported in Bai et al. (2008).

The data generating process has two basic building blocks, a $(r+m)$-dimensional vector of idiosyncratic variables, which is denoted by $Z_{i, t}=\left(Y_{i, t}^{\prime}, X_{i, t}^{\prime}\right)^{\prime}$, where $Y_{i, t}$ is $r \times 1$ while $X_{i, t}$ is $m \times 1$, and a $k$-dimensional vector of common factors, which is denoted by $F_{t}$. The grand vector containing all three variables is denoted $Z_{i, t}^{+}=\left(Z_{i, t}^{\prime}, F_{t}^{\prime}\right)^{\prime}$, and for later use we will also let $V_{i, t}=\left(X_{i, t}^{\prime}, F_{t}^{\prime}\right)^{\prime}$ denote the augmented $X_{i, t}$ vector.

The data generating process can be written in the following way

$$
\begin{aligned}
Y_{i, t}-\pi_{1 i}^{\prime} G_{t} & =b_{i}^{\prime} X_{i, t}+\lambda_{1 i}^{\prime} F_{t}+u_{1 i, t}, \\
\Delta X_{i, t}-\pi_{2 i}^{\prime} g_{t} & =\lambda_{2 i}^{\prime} \Delta F_{t}+u_{2 i, t}, \\
\Delta F_{t}-\pi_{3}^{\prime} g_{t} & =f_{t},
\end{aligned}
$$

where $G_{t}$ and $g_{t}$ are vectors of deterministic components such that $g_{t}=\Delta G_{t}$ with associated coefficients $\pi_{i}=\left(\begin{array}{lll}\pi_{1 i} & \pi_{2 i} & \pi_{3}\end{array}\right)$.

We further assume that the vector $u_{i, t}^{+}=\left(u_{1 i, t}^{\prime}, u_{2 i, t}^{\prime}, f_{t}^{\prime}\right)^{\prime}$ is a stationary linear process given by

$$
\begin{aligned}
u_{i, t}^{+} & =\left(\begin{array}{ccc}
\Gamma_{11 i}(L) & \Gamma_{12 i}(L) & 0 \\
\Gamma_{21 i}(L) & \Gamma_{22 i}(L) & 0 \\
0 & 0 & \Psi(L)
\end{array}\right)\left(\begin{array}{c}
\varepsilon_{1 i, t} \\
\varepsilon_{2 i, t} \\
\eta_{t}
\end{array}\right)=\left(\begin{array}{cc}
\Gamma_{i}(L) & 0 \\
0 & \Psi(L)
\end{array}\right)\left(\begin{array}{c}
\varepsilon_{i, t} \\
\eta_{t}
\end{array}\right) \\
& =\Gamma_{i}^{+}(L) \varepsilon_{i, t}^{+},
\end{aligned}
$$

where $\Psi(L)=I_{k}-\sum_{j=1}^{\infty} \Gamma_{33 j} L^{j}$ and $L$ is the lag operator. Similarly,

$$
\Gamma_{i}(L)=I_{(r+m)}-\sum_{j=1}^{\infty} \Gamma_{i j} L^{j} .
$$

Equations (1) to (4) constitute the triangular representation of the model. The rest of the assumptions can be summarized in the following way, where $M<\infty$ denotes a generic positive real number. 
Assumption 1. (i) $\eta_{t} \sim$ i.i.d. $\left(0, I_{k}\right)$ with finite fourth moments, (ii) $\sum_{j=0}^{\infty} j \cdot\left\|\Psi_{j}\right\|<M$, (iii) $r k(\Psi(1))=k$.

Assumption 2. (i) $\varepsilon_{i, t} \sim$ i.i.d. $\left(0, \Sigma_{i}\right)$ with finite eighth moments and

$$
\Sigma_{i}=\left(\begin{array}{cc}
\Sigma_{11 i} & \Sigma_{12 i} \\
\Sigma_{21 i} & \Sigma_{22 i}
\end{array}\right)=\operatorname{cov}\left(\varepsilon_{i, t}\right)
$$

(ii) $E\left(\varepsilon_{i, t} \varepsilon_{j, s}\right)=0$ for all $i \neq j$ and $t \neq s$, (iii) $\Gamma_{i}(L)$ fulfils the random coefficient and summability conditions of Phillips and Moon (1999, Assumptions 1 and 2), (iv) $r k\left(\Gamma_{i}(1)\right)=$ $r+m$.

Assumption 3. (i) $\Lambda_{i}=\left(\lambda_{1 i}, \lambda_{2 i}\right)^{\prime}$ is a random matrix such that $\left\|\Lambda_{i}\right\|<M$, (ii) $\bar{\Lambda}=$ $\frac{1}{N} \sum_{i=1}^{N} \Lambda_{i} \rightarrow \mathrm{E}\left(\Lambda_{i}\right)=\Lambda<M$ as $N \rightarrow \infty,($ iii $) r k(\bar{\Lambda})=k \leq r+m$.

Assumption 4. $\eta_{t}, \varepsilon_{i, t}$ and $\Lambda_{i}$ are mutually independent.

Assumptions 1, 2 and 4 imply that for any $i, \varepsilon_{i, t}^{+} \sim$ i.i.d. $\left(0, \Sigma_{i}\right)$ with

$$
\Sigma_{i}^{+}=\left(\begin{array}{cc}
\Sigma_{i} & 0 \\
0 & I_{k}
\end{array}\right)=\operatorname{cov}\left(\varepsilon_{i, t}^{+}\right)
$$

They also imply that $r k\left(\Gamma_{i}^{+}(1)\right)=r+m+k$. Under these assumptions, it is easy to see that the system has $r$ cointegrating relationships $\beta_{i}^{\prime} Z_{i, t}^{+}$, where by assumption

$$
\beta_{i}=\left(\begin{array}{ccc}
I_{r} & -b_{i}^{\prime} & -\lambda_{1 i}^{\prime}
\end{array}\right)^{\prime}
$$

is the cointegrating matrix.

Similar to the time series case considered by Cappuccio and Lubian (1996), given the triangular representation in (1) to (4), we can derive a Granger type representation theorem for a given panel member. This provides us with alternative model representations that are better suited for testing the hypothesis of no cointegration.

Theorem 1. Given the triangular representation in (1) to (4), $Z_{i, t}^{+}$is non-stationary with cointegration rank $r$.

(a) The $M A$ representation of $\Delta Z_{i, t}^{+}$is

$$
\Delta Z_{i, t}^{+}-\left(\pi_{i}^{*}\right)^{\prime} g_{t}=C_{i}(L) \varepsilon_{i, t}^{+},
$$

where $C_{i}(L)$ is given in the appendix, $r k\left(C_{i}(1)\right)=m+k$ and

$$
\pi_{i}^{*}=\left(\begin{array}{lll}
\pi_{1 i}+\pi_{2 i} b_{i}+\pi_{3}\left(\lambda_{1 i}+\lambda_{2 i} b_{i}\right) & \pi_{2 i}+\pi_{3} \lambda_{2 i} & \pi_{3}
\end{array}\right) .
$$


(b) The ARMA representation of $Z_{i, t}^{+}$is given by

$$
A_{i}(L)\left(Z_{i, t}^{+}-\left(\pi_{i}^{*}\right)^{\prime} g_{t}\right)=c_{i}(L) \varepsilon_{i, t}^{+},
$$

where $c_{i}(L)=\left|\Gamma_{i}^{+}(L)\right|$ is a scalar lag polynomial, and where the blocks of

$$
A_{i}(L)=\left(\begin{array}{ccc}
A_{11 i}(L) & A_{12 i}(L) & A_{13 i}(L) \\
A_{21 i}(L) & A_{22 i}(L) & A_{23 i}(L) \\
0 & 0 & A_{33 i}(L)
\end{array}\right)
$$

are given by

$$
\begin{aligned}
& A_{11 i}(L)=|\Psi(L)|\left|\Gamma_{22 i}(L)\right| \operatorname{adj}\left(\Gamma_{11 \cdot 2 i}(L)\right), \\
& A_{12 i}(L)=-|\Psi(L)|\left|\Gamma_{22 i}(L)\right| \operatorname{adj}\left(\Gamma_{11 \cdot 2 i}(L)\right)\left((1-L) \Gamma_{12 i}(L) \Gamma_{22 i}(L)^{-1}+b_{i}^{\prime}\right), \\
& A_{13 i}(L)=|\Psi(L)|\left|\Gamma_{22 i}(L)\right| \operatorname{adj}\left(\Gamma_{11 \cdot 2 i}(L)\right)\left((1-L) \Gamma_{12 i}(L) \Gamma_{22 i}(L)^{-1} \lambda_{2 i}^{\prime}-\lambda_{1 i}^{\prime}\right), \\
& A_{21 i}(L)=-|\Psi(L)| \operatorname{adj}\left(\Gamma_{22 i}(L)\right) \Gamma_{21 i}(L) \operatorname{adj}\left(\Gamma_{11 \cdot 2 i}(L)\right), \\
& A_{22 i}(L)=|\Psi(L)| \operatorname{adj}\left(\Gamma_{22 i}(L)\right)\left(\Gamma_{21 i}(L) \operatorname{adj}\left(\Gamma_{11 \cdot 2 i}(L)\right)\left((1-L) \Gamma_{12 i}(L) \Gamma_{22 i}(L)^{-1}+b_{i}^{\prime}\right)\right. \\
&\left.+(1-L)\left|\Gamma_{11 \cdot 2 i}(L)\right|\right), \\
& A_{23 i}(L)=-|\Psi(L)| \operatorname{adj}\left(\Gamma_{22 i}(L)\right)\left(\Gamma_{21 i}(L) \operatorname{adj}\left(\Gamma_{11 \cdot 2 i}(L)\right)\right. \\
&\left.\times\left((1-L) \Gamma_{12 i}(L) \Gamma_{22 i}(L)^{-1} \lambda_{2 i}^{\prime}-\lambda_{1 i}^{\prime}\right)+(1-L)\left|\Gamma_{11 \cdot 2 i}(L)\right| \lambda_{2 i}^{\prime}\right), \\
& A_{33 i}(L)=(1-L)\left|\Gamma_{22 i}(L) \| \Gamma_{11 \cdot 2 i}(L)\right| \operatorname{adj}(\Psi(L)), \\
& \text { with } \Gamma_{11 \cdot 2 i}(L)=\Gamma_{11 i}(L)-\Gamma_{12 i}(L) \Gamma_{22 i}(L)^{-1} \Gamma_{21 i}(L) .
\end{aligned}
$$

(c) $A_{i}(1)$ has reduced rank $r$ and can be decomposed as $A_{i}(1)=\alpha_{i}^{*} \beta_{i}^{\prime}$, where

$$
\alpha_{i}^{*}=\left(\begin{array}{c}
|\Psi(1)|\left|\Gamma_{22 i}(1)\right| \operatorname{adj}\left(\Gamma_{11 \cdot 2 i}(1)\right) \\
-|\Psi(1)| \operatorname{adj}\left(\Gamma_{22 i}(1)\right) \Gamma_{21 i}(1) \operatorname{adj}\left(\Gamma_{11 \cdot 2 i}(1)\right) \\
0
\end{array}\right) .
$$

(d) The vector ECM representation is

$$
A_{i}^{*}(L)\left(\Delta Z_{i, t}^{+}-\left(\pi_{i}^{*}\right)^{\prime} \Delta g_{t}\right)=-\alpha_{i}^{*} \beta_{i}^{\prime}\left(Z_{i, t-1}^{+}-\left(\pi_{i}^{*}\right)^{\prime} g_{t-1}\right)+c_{i}(L) \varepsilon_{i, t}^{+},
$$

where $A_{i}^{*}(L)=A_{i}^{+}(L)+A_{i}(1)$ with $A_{i}^{+}(L)$ satisfying $A_{i}(L)=A_{i}(1)+(1-L) A_{i}^{+}(L)$, $A_{i}^{+}(L)=\sum_{j=0}^{\infty} A_{i j}^{+} L^{J}$ and $A_{i j}^{+}=-\sum_{l=j+1}^{\infty} A_{i l}$.

(e) $\xi_{i, t}^{\prime}=\left(Z_{i, t}^{+}\right)^{\prime} \beta_{i}$ has the following representation

$$
\begin{aligned}
& \xi_{i, t}=\beta_{i}^{\prime}\left(\pi_{i}^{*}\right)^{\prime} G_{t}+\left(\begin{array}{cc}
\Gamma_{11 i}(L) & \Gamma_{12 i}(L)
\end{array}\right) \varepsilon_{i, t}^{+}, \\
& \Delta \xi_{i, t}-K_{i}(L)\left(\pi_{i}^{*}\right)^{\prime} g_{t}=-\beta_{i}^{\prime} \alpha_{i}^{*}\left(\xi_{i, t-1}-\beta_{i}^{\prime}\left(\pi_{i}^{*}\right)^{\prime} g_{t}\right)+J_{i}(L) \varepsilon_{i, t}^{+} \text {, }
\end{aligned}
$$

where $K_{i}(L)$ and $J_{i}(L)$ can be obtained as in Engle and Granger (1987). 
From the vector ECM representation given in (7) we can obtain the conditional ECM for $Y_{i, t}$ and the marginal ECM for $V_{i, t}$. Towards this end, let $\alpha_{i}=-A_{i}(0)^{-1} \alpha_{i}^{*}$ and $\tilde{A}_{i}^{*}(L)=$ $A_{i}(0)^{-1} A_{i}^{*}(L)$, where $\tilde{A}_{i}^{* *}(L)=\sum_{j=1}^{\infty} \tilde{A}_{i j}^{* *} L^{j}$ with $\tilde{A}_{i j}^{* *}=-\tilde{A}_{i j+1}^{*}$ such that

$$
\Delta Z_{i, t}^{+}-\tilde{A}_{i}^{*}(L)\left(\pi_{i}^{*}\right)^{\prime} \Delta g_{t}=\alpha_{i} \beta_{i}^{\prime}\left(Z_{i, t-1}^{+}-\left(\pi_{i}^{*}\right)^{\prime} g_{t-1}\right)+\tilde{A}_{i}^{* *}(L) \Delta Z_{i, t-1}^{+}+c_{i}(L) \varepsilon_{i, t}^{+} .
$$

Defining $B_{i}^{*}=\left(\Sigma_{12 i} \Sigma_{22 i}^{-1}+b_{i}^{\prime},-\Sigma_{12 i} \Sigma_{22 i}^{-1} \lambda_{2 i}^{\prime}+\lambda_{1 i}^{\prime}\right)$ and $\kappa_{i}=\left(I_{r},-B_{i}^{*}\right)$, the conditional ECM for $Y_{i, t}$ is given by

$$
\begin{aligned}
\Delta Y_{i, t}-\kappa_{i} \tilde{A}_{i}^{*}(L)\left(\pi_{i}^{*}\right)^{\prime} \Delta g_{t} & =B_{i}^{*} \Delta V_{i, t}+\kappa_{i} \alpha_{i} \beta_{i}^{\prime}\left(Z_{i, t-1}^{+}-\left(\pi_{i}^{*}\right)^{\prime} g_{t-1}\right)+\kappa_{i} \tilde{A}_{i}^{* *}(L) \Delta Z_{i, t-1}^{+} \\
& +c_{i}(L) \varepsilon_{1 \cdot 2 i, t}
\end{aligned}
$$

where $\varepsilon_{1 \cdot 2 i, t}=\varepsilon_{1 i, t}-\Sigma_{12 i} \Sigma_{22 i}^{-1} \varepsilon_{2 i, t}$, while the marginal models for $X_{i, t}$ and $F_{t}$ are

$$
\begin{aligned}
\Delta X_{i, t}-\tilde{A}_{2 i}^{*}(L)\left(\pi_{i}^{*}\right)^{\prime} \Delta g_{t} & =\alpha_{2 i} \beta_{i}^{\prime}\left(Z_{i, t-1}^{+}-\left(\pi_{i}^{*}\right)^{\prime} g_{t-1}\right)+\tilde{A}_{2 i}^{* *}(L) \Delta Z_{i, t-1}^{+}+c_{i}(L) \varepsilon_{2 i, t}^{*}, \\
\Delta F_{t}-\tilde{A}_{33 i}^{*}(L) \pi_{3}^{\prime} \Delta g_{t} & =A_{33 i}^{* *}(L) \Delta F_{t-1}+c_{i}(L) \eta_{t},
\end{aligned}
$$

where $\tilde{A}_{2 i}^{*}(L)$ and $\tilde{A}_{2 i}^{* *}(L)$ are the second rows of $\tilde{A}_{i}^{*}(L)$ and $\tilde{A}_{i}^{* *}(L)$, respectively, and where $\varepsilon_{2 i, t}^{*}=\varepsilon_{2 i, t}+\lambda_{2 i}^{\prime} \eta_{t}$.

Some remarks can be made here.

Remark 1. What this theorem shows is that alternative representations may lead naturally to alternative approaches to cointegration testing. In particular, while the triangular representation is better suited for developing residual-based tests, the vector ECM, and more precisely its factorization into conditional and marginal models, is more suitable for developing tests based on error correction.

Remark 2. If $\Gamma_{i}^{+}(L)$ is a unimodular matrix polynomial, the MA part in the vector ECM in (7) vanishes. Furthermore, if $\Gamma_{i}^{+}(L)$ is of order $p_{i}, A_{i}(L)$ is of order $q_{i} \leq(r+m+k-1) p_{i}$.

Remark 3. The common factor $F_{t}$ is by assumption strongly exogenous for $\beta_{i}$, see for example Urbain (1992) for weak and strong exogeneity conditions in this class of models. Similarly, $X_{i, t}$ is weakly exogenous for $\beta_{i}$ if $\alpha_{2 i}^{*}=0$, which will be the case when $\Gamma_{21 i}(1)=0$. It is strongly exogenous if in addition $\Gamma_{21 i}(L)=0$. The relevance of the two latter assumptions will be discussed later.

Remark 4. Depending on the specification of the deterministic component $g_{t}$, we can distinguish at least five variations of the ECM in (8) to (10). If $g_{t}=0$, henceforth referred to as Model 1, then there are no deterministic components present. If $\pi_{1 i}=0$, then $\beta_{i}^{\prime}\left(\pi_{i}^{*}\right)^{\prime}=0$ and hence $g_{t}$ do not appear in the error correction term. If in addition $g_{t}=(1, t)^{\prime}$, then a constant should be included, while if $g_{t}=\left(1, t, t^{2}\right)^{\prime}$, then a linear trend should also be included. 
These specifications are henceforth referred to as Models 2 and 3, respectively. Moreover, if $\pi_{1 i} \neq 0$, we have a constant restricted to the error correction term if $g_{t}=1$, henceforth referred to as Model 4, or an unrestricted constant and a linear trend in the error correction term if $g_{t}=(1, t)^{\prime}$, henceforth referred to as Model 5. Although higher order trend terms are certainly possible, such models are rarely used in practice, and we therefore restrict our attention to these five.

\section{Individual tests for no error correction}

In this section we show how the conditional ECM in (8) can be used as a basis for constructing cointegration tests. In particular, we propose two test statistics that are designed to test the null hypothesis that unit $i$ is not error correcting versus the alternative that it is error correcting. We begin by considering the baseline case with known factors, and then we show how the testing can be carried out in the more realistic case when $F_{t}$ is no longer observed.

\subsection{Observed factors}

Assumptions 1 to 4 are quite relaxed in the sense that even at the level of the individual unit, the models they imply are multivariate, which makes a full-blown system approach necessary. However, the purpose of this section is not to devise the most general test possible, but rather to derive tests that are simple, and easy to implement. This requires more assumptions.

Assumption 5. (i) $r=1$, (ii) $c_{i}(L)=c_{i}$ for some constant $c_{i}<M$, (iii) $X_{i, t}$ is weakly exogenous for $\alpha_{1 i}$ and $\beta_{i}$.

Remark 5. Assumption 5 implies that the $r$-dimensional conditional model in (8) can be written as a well-specified single equation, with no serial correlation and with the scalar coefficient $\alpha_{1 i}$ measuring the extent of the error correcting behavior in $Y_{i, t}$.

Under Assumption 5, and omitting any deterministic component for now, the conditional ECM in (8) reduces to

$$
\Delta Y_{i, t}=\alpha_{1 i} \beta_{i}^{\prime} Z_{i, t-1}^{+}+B_{11 i}(L) \Delta Y_{i, t-1}+B_{12 i}(L) \Delta X_{i, t}+B_{13 i}(L) \Delta F_{t}+\varepsilon_{1 \cdot 2 i, t},
$$

while the marginal models for $X_{i, t}$ and $F_{t}$ become

$$
\begin{aligned}
\Delta X_{i, t} & =B_{21 i}(L) \Delta Y_{i, t-1}+B_{22 i}(L) \Delta X_{i, t-1}+B_{23 i}(L) \Delta F_{t-1}+\varepsilon_{2 i, t}^{*}, \\
\Delta F_{t} & =B_{33 i}(L) \Delta F_{t-1}+\eta_{t},
\end{aligned}
$$

where the lag polynomials $B_{j l i}(L)$ are obtained by simply collecting the appropriate terms from (8) to (10). 
Assumptions 1 to 5 ensure that the following functional central limit theorem holds as $T \rightarrow \infty$

$$
\frac{1}{\sqrt{T}} \sum_{t=1}^{\lfloor s T\rfloor}\left(\begin{array}{c}
\varepsilon_{1 \cdot 2 i, t} \\
\varepsilon_{2 i, t}^{*} \\
\eta_{t}^{*}
\end{array}\right) \stackrel{w}{\longrightarrow} B_{i}
$$

where $s \in[0,1]$ and $B_{i}=\left(B_{1 i}, B_{2 i}^{\prime}, B_{3}^{\prime}\right)^{\prime}$ is a $(1+m+k)$-dimensional vector Brownian motion, which can be partitioned as $B_{i}=\left(B_{1 i}, B_{2 \cdot i}^{\prime}\right)^{\prime}$ with $B_{2 \cdot i}=\left(B_{2 i}^{\prime}, B_{3}^{\prime}\right)^{\prime}$ having dimension $m+k$. The covariance matrix of $B_{i}$ is given by

$$
\Xi_{i}=\left(\begin{array}{ccc}
\sigma_{i}^{2} & 0 & 0 \\
0 & \Sigma_{22 i}+\lambda_{2 i}^{\prime} \lambda_{2 i} & \lambda_{2 i}^{\prime} \\
0 & \lambda_{2 i} & I_{k}
\end{array}\right)=\operatorname{cov}\left(B_{i}\right)
$$

where $\sigma_{i}^{2}=\Sigma_{11 i}-\Sigma_{12 i} \Sigma_{22 i}^{-1} \Sigma_{21 i}$. Thus, $B_{i}=\Xi_{i}^{\frac{1}{2}} W_{i}$, where $W_{i}$ is a $(1+m+k)$-dimensional standard Brownian motion that is partitioned conformably with $B_{i}$. Furthermore, the longrun covariance matrix of $Z_{i, t}^{+}$is given by

$$
\Omega_{i}=\tilde{B}_{i}(1) \Xi_{i} \tilde{B}_{i}(L)^{\prime}=\Omega_{i}^{\frac{1}{2}}\left(\Omega_{i}^{\frac{1}{2}}\right)^{\prime}
$$

where the lag polynomial $\tilde{B}_{i}(L)$ is obtained from collecting the appropriate terms from (11) to $(13)$ and $\Omega_{i}^{\frac{1}{2}}=\tilde{B}_{i}(1) \Xi_{i}^{\frac{1}{2}}$.

For later reference it is useful to consider the continuous time regression of $W_{1 i}$, the first element of $W_{i}$, onto some vector $X_{i}$,

$$
W_{1 i}=P_{i}\left(X_{i}\right)^{\prime} X_{i}+Q_{X} W_{1 i}
$$

where

$$
P_{i}\left(X_{i}\right)=\left(\int X_{i} X_{i}^{\prime}\right)^{-1} \int X_{i} W_{1 i}=V\left(X_{i}\right) p_{i}\left(X_{i}\right)
$$

is the ordinary least squares (OLS) projection with $Q_{X} W_{1 i}$ being the associated projection error. For example, if $X_{i}=1$, then $P_{i}\left(X_{i}\right)=\int W_{1 i}$ in which case $Q_{1} W_{1 i}=W_{1 i}-\int W_{1 i}$ is the demeaned version of $W_{1 i}$.

As (11) makes clear, as long as $F_{t}$ is observed, the problem of testing the null of no error correction is equivalent to testing

$$
H_{0 i}: \alpha_{1 i}=0
$$

against

$$
H_{1 i}: \alpha_{1 i}<0 .
$$

The problem is that, unless one resorts to nonlinear techniques, this parameter is not easily estimated. One way to get around this is to assume that $\beta_{i}$ is known, and to estimate $\alpha_{1 i}$ using OLS. However, as shown by Boswijk (1994) and Zivot (2000), apart from the obvious 
drawback that $\beta_{i}$ is almost never known in practice, tests based on a prespecified $\beta_{i}$ are generally not similar and depend on nuisance parameters, even asymptotically.

As an alternative approach, note that (11) can be reparameterized as

$$
\begin{aligned}
\Delta Y_{i, t} & =\alpha_{1 i} Y_{i, t-1}+\gamma_{1 i}^{\prime} X_{i, t-1}+\gamma_{2 i}^{\prime} F_{t-1}+B_{11 i}(L) \Delta Y_{i, t-1}+B_{12 i}(L) \Delta X_{i, t} \\
& +B_{13 i}(L) \Delta F_{t}+\varepsilon_{1 \cdot 2 i, t},
\end{aligned}
$$

where $\gamma_{1 i}^{\prime}=-\alpha_{1 i} b_{i}^{\prime}$ and $\gamma_{2 i}^{\prime}=-\alpha_{1 i} \lambda_{1 i}^{\prime}$. The advantage of rewriting (11) in this way is that because $\gamma_{1 i}$ and $\gamma_{2 i}$ are unrestricted, the cointegrating vector is implicitly estimated under the alternative hypothesis. Hence, as long as we are not interested in $\beta_{i}$, all the parameters of (15) can be consistently estimated by simple OLS, which in turn suggests the OLS estimator of $\alpha_{1 i}$ as a natural candidate for constructing asymptotically similar tests of the null hypothesis of no error correction. In this section we propose two such tests, whose construction is described next.

One obvious candidate is the $t$-test. Suppose that the lag polynomial $B_{1 j i}(L)$ is of order $q_{i}$, and let

$$
W_{i, t}=\left(\Delta Y_{i, t-1}, \ldots, \Delta Y_{i, t-q_{i}}, \Delta X_{i, t}^{\prime}, \ldots, \Delta X_{i, t-q_{i}}^{\prime}, \Delta F_{t}^{\prime}, \ldots, \Delta F_{t-q_{i}}^{\prime}\right)^{\prime}
$$

denote the vector of stationary, first-differenced, regressors, while $V_{i, t}$ again denotes the vector of weakly exogenous non-stationary, level, variables, then (15) can be written as

$$
\begin{aligned}
\Delta Y_{i, t} & =\alpha_{1 i} Y_{i, t-1}+\gamma_{i}^{\prime} V_{i, t-1}+\Pi_{i}^{\prime} W_{i, t}+\varepsilon_{1 \cdot 2 i, t} \\
& =\alpha_{1 i} Y_{i, t-1}+\Phi_{i}^{\prime} S_{i, t}+\varepsilon_{1 \cdot 2 i, t},
\end{aligned}
$$

where $\Phi_{i}=\left(\gamma_{i}^{\prime}, \Pi_{i}^{\prime}\right)^{\prime}, S_{i, t}=\left(V_{i, t-1}^{\prime}, W_{i, t}^{\prime}\right)^{\prime}, \gamma_{i}=\left(\gamma_{1 i}^{\prime}, \gamma_{2 i}^{\prime}\right)^{\prime}$ and $\Pi_{i}$ is the vector stacking the coefficient vectors of the lag polynomials $B_{11 i}(L), B_{12 i}(L)$ and $B_{13 i}(L)$. This equation can in turn be written as

$$
\Delta\left(Q_{S} Y_{i, t}\right)=\alpha_{1 i}\left(Q_{S} Y_{i, t-1}\right)+Q_{S} \varepsilon_{1 \cdot 2 i, t},
$$

where again $Q_{S}$ is the OLS projection error operator, with

$$
Q_{S} Y_{i, t}=Y_{i, t}-\sum_{t=2}^{T} Y_{i, t-1} S_{i, t}^{\prime}\left(\sum_{t=2}^{T} S_{i, t} S_{i, t}^{\prime}\right)^{-1} S_{i, t}
$$

being the residual from projecting $Y_{i, t}$ onto $S_{i, t}$.

In this notation, the OLS estimator of $\alpha_{1 i}$ is given by

$$
\hat{\alpha}_{1 i}=\left(\sum_{t=2}^{T}\left(Q_{S} Y_{i, t-1}\right)^{2}\right)^{-1} \sum_{t=2}^{T} Q_{S} Y_{i, t-1} \Delta\left(Q_{S} Y_{i, t}\right),
$$


whose estimated variance is given by

$$
\operatorname{var}\left(\hat{\alpha}_{1 i}\right)=\hat{\sigma}_{i}^{2}\left(\sum_{t=2}^{T}\left(Q_{S} Y_{i, t-1}\right)^{2}\right)^{-1}
$$

where $\hat{\sigma}_{i}^{2}=\frac{1}{T} \sum_{t=2}^{T}\left(\Delta\left(Q_{S} Y_{i, t}\right)-\hat{\alpha}_{1 i}\left(Q_{S} Y_{i, t-1}\right)\right)^{2}$. The $t$-statistic for testing $H_{0 i}$ can now be written as

$$
\tau_{\hat{\alpha}_{1 i}}=\frac{\hat{\alpha}_{1 i}}{\sqrt{\operatorname{var}\left(\hat{\alpha}_{1 i}\right)}} .
$$

Another possibility is to follow Boswijk (1994), and to use a Wald statistic to test if $\alpha_{1 i}$ and $\gamma_{i}$ are jointly zero. In so doing, note that (16) can be rewritten as

$$
\Delta Y_{i, t}=\delta_{1 i}^{\prime} Z_{i, t-1}^{+}+\Pi_{i}^{\prime} W_{i, t}+\varepsilon_{1 \cdot 2 i, t}
$$

where $\delta_{1 i}=\left(\alpha_{1 i}, \gamma_{i}^{\prime}\right)^{\prime}$, or in terms of projection residuals,

$$
\Delta\left(Q_{W} Y_{i, t}\right)=\delta_{1 i}^{\prime}\left(Q_{W} Z_{i, t-1}^{+}\right)+Q_{W} \varepsilon_{1 \cdot 2 i, t}
$$

The Wald statistic for testing the restriction that $\delta_{1 i}=0$ is given by

$$
w_{\hat{\delta}_{1 i}}=\hat{\delta}_{1 i}^{\prime}\left(\operatorname{var}\left(\hat{\delta}_{1 i}\right)\right)^{-1} \hat{\delta}_{1 i}
$$

where

$$
\hat{\delta}_{1 i}=\left(\sum_{t=2}^{T} Q_{W} Z_{i, t-1}^{+}\left(Q_{W} Z_{i, t-1}^{+}\right)^{\prime}\right)^{-1} \sum_{t=2}^{T} Q_{W} Z_{i, t-1}^{+} \Delta\left(Q_{W} Y_{i, t}\right)
$$

is the OLS estimator of $\delta_{1 i}$, and

$$
\operatorname{var}\left(\hat{\delta}_{1 i}\right)=\hat{\sigma}_{i}^{2}\left(\sum_{t=2}^{T} Q_{W} Z_{i, t-1}^{+}\left(Q_{W} Z_{i, t-1}^{+}\right)^{\prime}\right)^{-1}
$$

is the associated variance.

The $t$-statistic $\tau_{\hat{\alpha}_{1 i}}$ and the Wald statistic $w_{\hat{\delta}_{1 i}}$ are the two test statistics considered in this paper. Their limiting distributions under the no error correction null are given in the following theorem.

Theorem 2. Under $H_{0 i}$ and Assumptions 1 to 5 , as $T \rightarrow \infty$

(a) $w_{\hat{\delta}_{1 i}} \stackrel{w}{\longrightarrow} D_{i, w}=p_{i}\left(W_{i}\right)^{\prime} P_{i}\left(W_{i}\right)$,

(b) $\tau_{\hat{\alpha}_{1 i}} \stackrel{w}{\longrightarrow} D_{i, \tau}^{\Omega}=\frac{d_{i}}{\sqrt{D_{i}}}$, 
where $p_{i}(\cdot)$ and $P_{i}(\cdot)$ are defined in (14),

$$
\begin{aligned}
D_{i} & =\sigma_{i}^{2} \omega_{11 \cdot 2 i}^{-2} V\left(U_{i}\right) \\
& +\sigma_{i}^{2} \omega_{11 \cdot 2 i}^{-2} \omega_{11 i} V\left(U_{i}\right)\left(\rho_{i}^{\prime}\left(\Omega_{22 i}^{\prime}\right)^{-1} P_{i}\left(W_{2 \cdot i}\right)+V\left(W_{2 \cdot i}\right) p_{i}\left(W_{2 \cdot i}\right)^{\prime} \Omega_{22 i}^{-1} \rho_{i}\right) \\
& +\sigma_{i}^{2} \omega_{11 \cdot 2 i}^{-2} \omega_{11 i}^{2} \rho_{i}^{\prime}\left(\Omega_{22 i}^{\prime}\right)^{-1}\left(V\left(W_{2 \cdot i}\right)+P_{i}\left(W_{2 \cdot i}\right) V\left(U_{i}\right) V\left(W_{2 \cdot i}\right) p_{i}\left(W_{2 \cdot i}\right)^{\prime}\right) \Omega_{22 i}^{-1} \rho_{i}, \\
d_{i} & =\sigma_{i} \omega_{11 \cdot 2 i}^{-1} P_{i}\left(U_{i}\right)+\omega_{11 \cdot 2 i}^{-1} \omega_{11 i} \rho_{i}^{\prime}\left(\Omega_{22 i}^{\prime}\right)^{-1} P_{i}\left(W_{2 \cdot i}\right)\left(P_{i}\left(U_{i}\right)-1\right),
\end{aligned}
$$

with $\omega_{11 \cdot 2 i}, \omega_{11 i}, \Omega_{22 i}$ and $\rho_{i}$ depending on the parameters of $\Omega_{i}^{\frac{1}{2}}$, as defined in the appendix.

The asymptotic distribution of $\tau_{\hat{\alpha}_{1 i}}$ simplifies substantially if $X_{i, t}$ is strongly exogenous.

Assumption 6. $X_{i, t}$ is strongly exogenous for $\alpha_{1 i}$ and $\beta_{i}$.

This is shown in the following corollary.

Corollary 1. Under Assumption 6 and the conditions of Theorem 2, as $T \rightarrow \infty$,

$$
\tau_{\hat{\alpha}_{1 i}} \stackrel{w}{\longrightarrow} D_{i, \tau}=\frac{P_{i}\left(U_{i}\right)}{\sqrt{V\left(U_{i}\right)}} .
$$

Theorem 2 shows that the distribution of $w_{\hat{\delta}_{1 i}}$ as $T \rightarrow \infty$ is nuisance parameter free and only depends on $m+k$, the number of non-stationary exogenous variables in the system. By contrast, the distribution of $\tau_{\hat{\alpha}_{1 i}}$ depends on several nuisance parameters, and although these vanish under Assumption 6, strong exogeneity is quite restrictive. Fortunately, as Zivot (2000) points out relying on results obtained by for example Saikkonen (1991), there is a simple modification available that eliminates the nuisance parameters that are there under Assumption 5 (iii). The idea is to model these parameters by making the lag polynomial $B_{12 i}(L)$ double-sided, as in

$$
B_{12 i}\left(L+L^{-1}\right)=\sum_{j=-\infty}^{\infty} B_{12 i j} L^{j},
$$

where $L^{-1}$ is the lead operator, which in in turn requires augmenting (16) not only by the lags, but also by the leads of $\Delta X_{i, t}$. If the number of leads is large enough, then the asymptotic distribution of the resulting test statistic is given in Corollary 1.

In this sense, the results in Theorem 1 are basically the same as those provided by Banerjee et al. (1998) and Boswijk (1994) for the pure time series case. The proof is therefore very similar. The difference lies with the presence of $F_{t}$, which has two effects. One is that the number of unit roots increases from $1+m$ to $1+m+k$, which is reflected through $W_{3}$ in the asymptotic distribution of the test. The second effect is that the test statistics across units are no longer independent of each other, although the degree of the dependence between all pairs of units is the same. 
In the presence of nonzero deterministic constant and trend terms, as in Models 2 to 5 , the above theorem needs to be modified in order to obtain similar tests. This requires replacing $U_{i}$ in (a) and $W_{i}$ in (b) by their appropriately detrended counterparts. Specifically, $U_{i}$ and $W_{i}$ should be demeaned in Model 1, and demeaned and detrended in Model 2. The $t$-test cannot be used in Models 4 and 5, and so for these models there is only the Wald test. In Model $4, W_{i}$ is replaced by $\left(W_{i}^{\prime}, 1\right)^{\prime}$, while in Model $5, W_{i}$ is replaced by $\left(W_{i}^{\prime}, 1, s\right)^{\prime}$, where $s$ is the limiting trend function, see Boswijk (1994).

Furthermore, under the alternative hypothesis of cointegration, $\tau_{\hat{\alpha}_{1 i}} \rightarrow-\infty$ whereas

$w_{\hat{\delta}_{1 i}} \rightarrow \infty$ as $T \rightarrow \infty$, suggesting that the tests are consistent. A proof of this is provided by Boswijk (1994).

\subsection{Unobserved factors}

So far we have assumed $F_{t}$ to be observed, an assumption which is generally not true. To account for this, in a recent unit root paper Bai and $\mathrm{Ng}$ (2004) propose using the method of principal components to estimate $F_{t}$, and then to use this estimate in place of $F_{t}$ in the subsequent analysis. This approach has proven very fruitful, and has also been extended to the case of cointegration, see for example Bai et al. (2008), Banerjee and Carrio-i-Silvestre (2007), Gengenbach et al. (2006) and Westerlund (2008). The problem with this approach is that, regardless of whether one considers unit roots or cointegration, the analysis must be carried out in steps, which means that the estimation error from one step is imported in subsequent steps.

As a response to this, Pesaran (2007) proposes a joint approach, which is based on using cross-sectional averages of the observed variables as proxies for the unobserved common factors. Apart from the advantage that it eliminates the need for a two-step estimation procedure, this approach fits very well with the parametric flavor of our conditional ECM, and it will therefore be used in this paper.

Part (b) of Theorem 1 implies that $Z_{i, t}$ can be written as

$$
Z_{i, t}=\Lambda_{i} F_{t}+E_{i, t}
$$

where $\Lambda_{i}$ is the $(1+m) \times k$ matrix of factor loadings, and where $E_{i, t}$ is a vector representing the idiosyncratic component of $Z_{i, t}$. Denoting by $\bar{Z}_{t}, \bar{\Lambda}$ and $\bar{E}_{t}$ the cross-sectional averages of $Z_{i, t}, \Lambda_{i}$ and $E_{i, t}$, respectively, it is clear that

$$
\bar{Z}_{t}=\bar{\Lambda} F_{t}+\bar{E}_{t}
$$

which, via Assumption 3 (iii) and the fact that $E_{i, t}$ is cross-sectionally independent, suggests that $F_{t}$ can be written as

$$
F_{t}=\left(\bar{\Lambda}^{\prime} \bar{\Lambda}\right)^{-1} \bar{\Lambda}^{\prime} \bar{Z}_{t}+\left(\bar{\Lambda}^{\prime} \bar{\Lambda}\right)^{-1} \bar{\Lambda}^{\prime} \bar{E}_{t}=\left(\bar{\Lambda}^{\prime} \bar{\Lambda}\right)^{-1} \bar{\Lambda}^{\prime} \bar{Z}_{t}+O_{p}\left(\frac{1}{\sqrt{N}}\right) .
$$


The implication is that the common factors can be approximated by the cross-sectional averages $\bar{Z}_{t}$, and that the resulting approximation error should become negligible as $N \rightarrow \infty$. Following this argument, we propose using $\bar{Z}_{t}$ to approximate $F_{t}$. In so doing, it is convenient to let $\tilde{W}_{i, t}, \tilde{V}_{i, t}$ and $\tilde{Z}_{i, t}^{+}$denote $W_{i, t}, V_{i, t}$ and $Z_{i, t}^{+}$, respectively, with $\bar{Z}_{t}$ in place of $F_{t}$. Starting with (16) the approximate test regression can now be written as

$$
\Delta Y_{i, t}=\alpha_{1 i} Y_{i, t-1}+\Phi_{i}^{\prime} \tilde{S}_{i, t}+\tilde{\varepsilon}_{1 \cdot 2 i, t}
$$

or equivalently,

$$
\Delta\left(Q_{\tilde{S}} Y_{i, t}\right)=\alpha_{1 i}\left(Q_{\tilde{S}} Y_{i, t-1}\right)+Q_{\tilde{S}} \tilde{\varepsilon}_{1 \cdot 2 i, t},
$$

where the error $\tilde{\varepsilon}_{1 \cdot 2 i, t}$ depends on the accuracy of the approximation. Nevertheless, by regressing $\Delta\left(Q_{\tilde{S}} Y_{i, t}\right)$ on $Q_{\tilde{S}} Y_{i, t-1}$, we obtain another OLS estimator of $\alpha_{1 i}$, which we will henceforth denote by $\tilde{\alpha}_{1 i}$. The associated $t$-statistic of $H_{0 i}$ can be written in an obvious notation as

$$
\tau_{\tilde{\alpha}_{1 i}}=\frac{\tilde{\alpha}_{1 i}}{\sqrt{\operatorname{var}\left(\tilde{\alpha}_{1 i}\right)}}
$$

while the Wald statistic can be written as

$$
w_{\tilde{\delta}_{1 i}}=\left(\tilde{\delta}_{1 i}\right)^{\prime}\left(\operatorname{var}\left(\tilde{\delta}_{1 i}\right)\right)^{-1} \tilde{\delta}_{1 i}
$$

where $\tilde{\delta}_{1 i}$ and $\operatorname{var}\left(\tilde{\delta}_{1 i}\right)$ are defined just as in Section 3.1 but with $Q_{\tilde{W}}$ in place of $Q_{W}$.

Theorem 3 provides the limiting null distributions of these test statistics.

Theorem 3. Under the conditions of Theorem 2, as $N, T \rightarrow \infty$,

(a) $w_{\tilde{\delta}_{1 i}} \stackrel{w}{\longrightarrow} D_{i, w}$,

(b) $\tau_{\tilde{\alpha}_{1 i}} \stackrel{w}{\longrightarrow} \tilde{D}_{i, \tau}^{\Omega}=\frac{\tilde{d}_{i}}{\sqrt{\tilde{D}_{i}}}$,

where $\tilde{d}_{i}$ and $\tilde{D}_{i}$ are defined analogously to $d_{i}$ and $D_{i}$ but depending on the parameters of $\tilde{\Omega}_{i}^{\frac{1}{2}}$, as defined in the appendix.

Theorem 3 shows that the asymptotic distributions of $w_{\tilde{\delta}_{1 i}}$ is the same as that of $w_{\hat{\delta}_{1 i}}$ provided in Theorem 2, which is based on observed factors. The limiting distribution of $\tau_{\tilde{\alpha}_{1 i}}$ is similar to that of $\tau_{\hat{\alpha}_{1 i}}$ but depending on different nuisance parameter due to the approximation of $F_{t}$ by $\bar{Z}_{t}$. The difference is that Theorem 2 only requires that $T \rightarrow \infty$. If $F_{t}$ is not observed, we require $N \rightarrow \infty$ as well to ensure that $\bar{Z}_{t}$ provides a sufficiently good approximation for $F_{t}$.

Similarly to the case of observed factors, if $X_{i, t}$ is strongly exogenous the asymptotic distribution of $\tau_{\tilde{\alpha}_{1 i}}$ simplifies and is the same as that of $\tau_{\hat{\alpha}_{1 i}}$. This is shown in Corollary 2 .

Corollary 2. Under Assumption 6 and the conditions of Theorem 3, as $N, T \rightarrow \infty$,

$$
\tau_{\hat{\alpha}_{1 i}} \stackrel{w}{\longrightarrow} D_{i, \tau} .
$$




\subsection{Critical values}

As in the simple case with cross-sectionally independent units, our tests are one-sided. The $t$-test is left-tailed, while the Wald test is right-tailed. The difference is that in our case the asymptotic test distribution, and hence also the simulation of the critical values, is complicated by the dependence across $i$. However, conditional on $W_{3}$, the Brownian motion associated with $F_{t}$, the random variables $D_{1, w}, \ldots, D_{N, w}$, are identically and independently distributed for all values of $N$. We say that $D_{1, w}, \ldots, D_{N, w}$ form an exchangeable sequence, similar to for example Pesaran (2007) and Gregoir (2005). Thus, since $D_{i, w}$ is the same for all $N$, we can just as well set $N=1$ in the simulations, a finding also confirmed by our results. The same argument applies to $D_{i, \tau}$. However, this is only valid for the limiting distribution of the $t$-test under strong exogeneity of $X_{i, t}$, or if an appropriate correction is employed to remove the nuisance parameter dependence. Otherwise, the individual test statistics are not identically distributed across $i$.

The simulated critical values at the $1 \%, 5 \%$ and $10 \%$ significance levels are reported in Table 1 for the $t$-test, and in Table 2 for the Wald test. These are based on making 1,000,000 draws from the limiting test distributions, with normal random walks of length $T=1,000$. The results are reported for all five deterministic model specifications, and for $m=1, \ldots, 5$.

\section{Panel tests for no error correction}

In this section we build on the results of Section 3, and show how these can be used to construct pooled tests for the null of no error correction at the overall panel level. As an example, we will consider the $t$-statistic in the most simple case with known factors.

\subsection{The tests}

There are many ways in which one can combine a set of individual test statistics into a pooled test. The by far most common way is to follow Im et al. (2003) and to take the average, which for the $t$-statistic in case of known factors amounts to computing

$$
\bar{\tau}_{\hat{\alpha}_{1}}=\frac{1}{N} \sum_{i=1}^{N} \tau_{\hat{\alpha}_{1 i}} .
$$

This is a test of the null of no error correction against the alternative that there is a non-

vanishing fraction of error correcting units. Formally, the null and alternative hypotheses are formulated as

$$
H_{0}: \alpha_{1 i}=0 \text { for all } i
$$

against

$$
H_{1}: \alpha_{1 i}<0 \text { for } i=1, \ldots, N_{1} \text { with } \frac{N_{1}}{N} \rightarrow \delta>0
$$


as $N_{1}, N \rightarrow \infty$. However, due to the dependence across $i$, in our case it is not possible to follow the usual practice in applying a central limit theorem to obtain a normal distribution for $\sqrt{N}$ times $\bar{\tau}_{\hat{\alpha}_{1}}$.

One possibility is to look directly at the average. Following similar arguments as Pesaran (2007), because $D_{1, \tau}, \ldots, D_{N, \tau}$ are identically and independently distributed given $W_{3}$, a law of large numbers applies to the conditional average of these random variables. That is, we have that as $N \rightarrow \infty$

$$
\bar{D}_{\tau}=\frac{1}{N} \sum_{i=1}^{N} D_{i, \tau} \stackrel{p}{\longrightarrow} \mathrm{E}\left(D_{\tau} \mid W_{3}\right),
$$

where the $i$ index in the expectation has been suppressed because all $D_{i, \tau}$ have the same conditional expectation. Thus, unconditionally the average converges to some random distribution. However, unless $\tau_{\hat{\alpha}_{1 i}}$ has finite moments for all $N$ and $T$, this distribution is not necessarily the same as the one that applies to $\bar{\tau}_{\hat{\alpha}_{1}}$.

In order to get around this technical difficulty, we follow Pesaran (2007) and base our pooled test on a truncated version of $\tau_{\hat{\alpha}_{1 i}}$. Because this test has finite moments by construction, the associated cross-sectional average converges to the same asymptotic distribution as $\bar{D}_{\tau}$.

The truncated statistic is defined as

$$
\tau_{\hat{\alpha}_{1 i}}^{*}=\left\{\begin{array}{cll}
K_{l} & \text { if } & \tau_{\hat{\alpha}_{1 i}} \leq K_{l} \\
\tau_{\hat{\alpha}_{1 i}} & \text { if } & K_{l}<\tau_{\hat{\alpha}_{1 i}}<K_{u} \\
K_{u} & \text { if } & \tau_{\hat{\alpha}_{1 i}} \geq K_{u}
\end{array}\right.
$$

where the thresholds $K_{l}$ and $K_{u}$ are such that the probability of observing $\tau_{\hat{\alpha}_{1 i}} \leq K_{l}$ and $\tau_{\hat{\alpha}_{1 i}} \geq K_{u}$ is sufficiently small. In particular, by using the normal approximation of $\tau_{\hat{\alpha}_{1 i}}$, $K_{l}=\mathrm{E}\left(D_{\tau}\right)-\Phi^{-1}\left(1-\frac{\varepsilon}{2}\right) \sqrt{\operatorname{var}\left(D_{\tau}\right)}$ and $K_{u}=\mathrm{E}\left(D_{\tau}\right)+\Phi^{-1}\left(1-\frac{\varepsilon}{2}\right) \sqrt{\operatorname{var}\left(D_{\tau}\right)}$, where $\varepsilon>0$ is a small number, while $\Phi$ is the standard normal cumulative distribution function.

The corresponding truncated version of $\bar{\tau}_{\hat{\alpha}_{1}}$ is given by

$$
\bar{\tau}_{\hat{\alpha}_{1}}^{*}=\frac{1}{N} \sum_{i=1}^{N} \tau_{\hat{\alpha}_{1 i}}^{*} .
$$

Making use of Theorem 2, it is not difficult to see that as $T \rightarrow \infty$

$$
\bar{\tau}_{\hat{\alpha}_{1}}^{*} \stackrel{w}{\longrightarrow} \bar{D}_{\tau}^{*}=\frac{1}{N} \sum_{i=1}^{N} D_{i, \tau}^{*},
$$

where

$$
D_{i, \tau}^{*}=\left\{\begin{array}{cll}
K_{l} & \text { if } \quad D_{i, \tau} \leq K_{l} \\
D_{i, \tau} & \text { if } \quad K_{l}<D_{i, \tau}<K_{u} \\
K_{u} & \text { if } \quad D_{i, \tau} \geq K_{u}
\end{array}\right.
$$

But all moments of $D_{i, \tau}^{*}$ exist, so by conditioning on $W_{3}$, as $N \rightarrow \infty$

$$
\bar{D}_{\tau}^{*} \stackrel{p}{\longrightarrow} \mathrm{E}\left(D_{\tau}^{*} \mid W_{3}\right)
$$


where

$$
\begin{aligned}
\mathrm{E}\left(D_{\tau}^{*} \mid W_{3}\right) & =K_{l} \cdot \operatorname{Prob}\left(D_{\tau} \leq K_{l} \mid W_{3}\right)+K_{u} \cdot \operatorname{Prob}\left(D_{\tau} \geq K_{u} \mid W_{3}\right) \\
& +\mathrm{E}\left(D_{\tau} \mid W_{3}, K_{l}<D_{\tau}<K_{u}\right) \rightarrow \mathrm{E}\left(D_{\tau} \mid W_{3}\right)
\end{aligned}
$$

as $K_{l}, K_{u} \rightarrow \infty$, and so we get the same result as for $\bar{D}_{\tau}$. This suggests that $\bar{\tau}_{\hat{\alpha}_{1}}^{*}$ can be used for the test of $H_{0}$ versus $H_{1}$. Another possibility is to use $\bar{w}_{\hat{\delta}_{1}}^{*}$, the average of the truncated Wald test statistics.

\subsection{Critical values}

The above results show that if $K_{l}, K_{u} \rightarrow \infty, \bar{\tau}_{\hat{\alpha}_{1}}^{*}$ converges to a distribution that only depends on number of non-stationary variables in the system. With $K_{l}$ and $K_{u}$ finite, however, then there is not just this dependence, but also a dependence on the specific threshold values. Similarly, if $N$ is finite, then there is also a dependence on the size of the cross-section. The generation of the critical values has to account for all these dependencies.

We begin by simulating values of $\mathrm{E}\left(D_{\tau}\right)$ and $\operatorname{var}\left(D_{\tau}\right)$ for all five deterministic model specifications, and for $m=1, \ldots, 5$. These are needed in order to compute $K_{l}$ and $K_{u}$. Just as in Section 3.3 we make 1,000,000 draws from the limiting test distribution, with normal random walks of length $T=1,000$. The results for the $t$-test are reported in Table 1 , while the results for the Wald test are reported in Table 2.

The next step is to simulate $N$-tuples $D_{1, \tau}^{*}, \ldots, D_{N, \tau}^{*}$ using $\varepsilon=\frac{1}{10^{6}}$, and the first-step moments to compute $K_{l}$ and $K_{u}$. The average is then taken, which yields one simulated value of $\bar{D}_{\tau}^{*}$. By repeating this exercise 10,000 times, we obtain the simulated distribution of $\bar{D}_{\tau}^{*}$. The critical values at the $1 \%, 5 \%$ and $10 \%$ levels are reported in Table 3 for the $t$-test and in Table 4 for the Wald test, in which case $\bar{D}_{\tau}^{*}$ is replaced by $\bar{D}_{w}^{*}$, the average of the truncated Wald test distributions.

\section{Monte Carlo simulations}

In this section we report the findings of a small set of simulations. We do not intend to give a comprehensive account of all the merits and drawbacks of the tests, but rather we want to convey a rough idea of their relative performance, also when compared to some of the more conventional tests from the literature.

The data generating conditional ECM is given by

$$
\Delta Y_{i, t}=\alpha_{1}\left(Y_{i, t-1}-X_{i, t-1}-\iota_{2}^{\prime} F_{t-1}\right)+\Delta X_{i, t}+B_{13 i} \Delta F_{t}+\varepsilon_{1 \cdot 2 i, t},
$$

while the marginal models for $X_{i, t}$ and $F_{t}$ are generated as

$$
\begin{aligned}
\Delta X_{i, t} & =B_{23 i} \Delta F_{t-1}+\varepsilon_{2 i, t}^{*}, \\
\Delta F_{t} & =\eta_{t},
\end{aligned}
$$


where the elements of $B_{23 i}$ and $B_{13 i}$ are drawn from $N(1,1)$, while $\iota_{2}=(1,1)^{\prime}$ is a twodimensional vector of ones. Thus, in this setup $X_{i, t}$ is a scalar, while $F_{t}$ is two-dimensional. For simplicity, we assume that there are no deterministic components in the data generating process, and that there is a common error correction parameter $\alpha_{1}$, which is equal to zero under the null hypothesis, and equal to -0.05 under the alternative.

The results are organized in four parts depending on whether there is any serial correlation present or not. If there is no serial correlation, then $\varepsilon_{1 \cdot 2 i, t}, \varepsilon_{2 i, t}^{*}$ and $\eta_{t}$ are drawn from the standard normal distribution, while if there is serial correlation, then one of these errors is specified as a first-order autoregressive (AR) process with standard normal innovations, and a common AR coefficient of magnitude 0.5, while the remaining two errors are again drawn from the standard normal distribution.

All experiments are based on generating 5,000 panels with $N$ individual and $T+50$ time series observations, where the first 50 observations for each series are discarded in order to attenuate the effect of the initial conditions, which are all set to zero.

For comparison, the error correction tests of Westerlund (2007) are also simulated. Two are based on the group mean, or between, principle and are denoted $G_{\tau}$ and $G_{\rho}$, while the corresponding panel, or within, type statistics are denoted $P_{\tau}$ and $P_{\rho}$. Analogous to $\bar{\tau}_{\hat{\alpha}_{1}}^{*}, G_{\tau}$ and $P_{\tau}$ are constructed as $t$-ratios, while $G_{\rho}$ and $P_{\rho}$ are coefficient type statistics.

The problem with these tests is that they are based on assuming cross-sectional independence, as explained earlier, and are therefore not expected to work in a setup as general as this one. Therefore, for better comparability, we follow the suggestion of Gengenbach et al. (2006), and run the tests on the defactored data. Specifically, we begin by estimating separately the common component of $X_{i, t}$ and $Y_{i, t}$ using the method of Bai and $\mathrm{Ng}$ (2004), which involves applying the principal components method to the variables in their first differences. The estimated common component is then removed, and the defactored data are cumulated back to levels again. The number of factors are determined using the $I C_{1}$ information criterion of Bai and $\mathrm{Ng}$ (2002) with a maximum of five factors.

For the number of lags and leads to use in the conditional ECM, we used the Schwarz Bayesian information criterion, which facilitates a data dependent choice. Consistent with the results of $\mathrm{Ng}$ and Perron (1995), the maximum number of lags and leads is permitted to grow with $T$ at rate $4\left(\frac{T}{100}\right)^{2 / 9}$. The same rate is used for picking the bandwidth needed for constructing $G_{\rho}$ and $P_{\rho}$. Also, for better comparability across all tests, we do not consider Models 4 and 5 when the deterministic constant and trend terms are restricted to the error correction term. All tests are performed at the $5 \%$ significance level, and all powers are adjusted for size.

The results for the case with no deterministic components are reported in Table 5. The first thing to note is the relative performance of the new $t$-tests, which is very good. This is 
especially true when the data are serially correlated, in which case there are only one other test with roughly the same performance as ours, $G_{\tau}$. The overall best performance is obtained by using the individual $\tau_{\hat{\alpha}_{1 i}}$ and $\tau_{\tilde{\alpha}_{1 i}}$ tests, which seem to maintain the nominal level very well in all cases considered. At the other end of the scale we have the $\bar{w}_{\hat{\delta}_{1}}$ test, which generally suffers from severe distortions, even if it is based on the true factors.

Pesavento (2004) reports some results for the original Wald test of Boswijk (1994), and find it to be oversized when the serial correlation is of the positive AR type considered here. The overall poor performance of the new Wald tests is therefore not very surprising. On the other hand, unreported results suggest that the relative performance of these tests is much improved if the serial correlation is of the negative MA type, which is also what Pesavento (2004) finds in her simulation study. In any case, the size distortions generally decrease substantially as $T$ increases, which corroborates our asymptotic results. ${ }^{1}$

Among the different versions of the new tests considered, the best size accuracy is not surprisingly obtained by using the true factors. The tests based on using the cross-sectional averages of the observed data as proxies for the factors are, however, almost as accurate, and perform only slightly less well. Thus, the approximation seem to be effective even when $N$ is as small as 10. The defactored versions of the tests of Westerlund (2007) also seem to perform quite well, which is in agreement with consistency of the principal components method, as shown by Bai and $\mathrm{Ng}$ (2004). However, although improving in $N$, we also see that the size accuracy is basically unaffected by $T$, which is unexpected because theoretically the precision of the principal components estimator should get better as $T$ grows.

Consider next the results reported in Table 5 for the power of the tests, which can be summarized as follows. Firstly, the power increases rapidly as $T$ and $N$ increase, which is presumably a reflection of the consistency of the tests. Secondly, the Westerlund (2007) tests generally suffer from poor power, especially when $\varepsilon_{1 \cdot 2 i, t}$ is serially correlated, in which case the power is only rarely in excess of the size. The $G_{\rho}$ test suffers most, and can actually be less powerful than some of the individual tests. Thirdly, as expected, the power of the new tests is generally greatly improved by pooling. Similarly, the tests based on the true factors are generally more powerful than those based on the cross-sectional averages of the observed data.

The results for the models with a constant, and constant and trend reported in Tables 6 and 7, respectively, are very similar to those reported in Table 5. Nevertheless, there are still a few differences that are noteworthy. One difference is the magnitude of the size distortions, which has a slight tendency to increase as more deterministic components are added. Similarly, we see that inclusion of more deterministic components reduces the power

\footnotetext{
${ }^{1}$ One possibility here is to follow Palm et al. (2007), and to use bootstrap methods to eliminate the size distortions of the Wald test.
} 
of the tests, especially for the individual ones. Another difference is that the relative power of the Wald tests is generally much higher in Tables 6 and 7 than in Table 5 .

We also examined the effects of a violation of the weak exogeneity assumption. We used the same data generating process as before but this time we allowed the equation for $\Delta X_{i, t}$ to be error correcting. The results, which are not reported but available from the corresponding author upon request, conforms well with our expectations. In particular, while the size of the tests is not effected, the power can be very low in cases when it is mainly $\Delta X_{i, t}$ that is error correcting. Thus, even though the tests continue to perform well in some setups, in general we need the weak exogeneity assumption to ensure that they work properly. ${ }^{2}$

The above results are all based on the truncated panel statistics. We carried out the same simulations for their non-truncated versions, and obtained identical results. In fact, the two types of statistics differ only for very small values of $T$, and are basically indistinguishable for $T>20$. Thus, although little is gained in the present case, the truncation of the extreme test statistics seem to pay out when $T$ is very small. This effect is particularly strong when the number parameters of the underlying ECM regressions is large.

\section{Empirical Applications}

In this section we present two empirical applications of the tests developed in this paper. The first is concerned with the Fisher effect, while the second is concerned with the monetary exchange rate model.

\subsection{The Fisher effect}

There are very few theoretical economic relationships with as much intuitive appeal as the Fisher effect, which states that a one-time permanent shock in monetary variables has no long-run effect on the real economy. A simple implication of this theory is that changes in inflation should be reflected fully in subsequent movements of the nominal interest rate, thus leaving the real interest rate constant over time. Yet, oddly, for a theory so widely accepted, the postulated long-run relationship between inflation and nominal interest rates has proven extremely difficult to establish empirically. In fact, most studies are unable to reject the null hypothesis of no cointegration between inflation and nominal interest rates.

Westerlund (2008) argues that this lack of empirical support can be partly explained by the poor precision of the routinely applied time series approach, and that the use of panel data can produce more accurate tests. Consistent with this story, drawing upon a panel of 20 OECD countries between the first quarter of 1980 and the fourth quarter of 2004, the author shows that while the null hypothesis of no cointegration cannot be rejected at

\footnotetext{
${ }^{2}$ Zivot (2000) examines the performance of the time series tests of Banerjee et al. (1998) and Boswijk (1994), and reach the same conclusions.
} 
conventional significance levels when using data on individual countries, panel testing leads to a safe rejection. Low power in the tests is therefore one possible explanation for why cointegration has been so difficult to find.

Our findings suggest that there is an alternative interpretation of these results. Namely, that inflation and nominal interest rates are cross-sectionally correlated via the presence of non-stationary common factors, which then invalidates the use of conventional critical values. ${ }^{3}$ Thus, according to this view, it is the factors, and not a lack of power, that make the tests unable to reject the no cointegration null at the individual country level. ${ }^{4}$

In this section, we therefore apply our new tests to the same data to reevaluate the cointegration test results reported by Westerlund (2008). In so doing, we will assume that his unit root test results hold, and hence that the rates of inflation and nominal interest are non-stationary. Hence, in this application $Y_{i, t}=i_{i, t}$ and $X_{i, t}=\pi_{i, t}$, where $i_{i, t}$ is the nominal interest rate for country $i$ in quarter $t$, while $\pi_{i, t}$ is inflation.

The tests are constructed in the same way as in Section 5, using the Schwarz Bayesian information criterion with the same maximum to determine the number of lags and leads. One difference in comparison to the simulations is that the common factors are no longer observed, which means that we cannot evaluate the tests at the true factors. Therefore, as a feasible alternative, in this section we consider replacing the factors by their first differenced and cumulated principal components estimates, which are consistent even if the factors are non-stationary, see Bai and Ng (2004). In agreement with the so-called full Fisher effect, the estimation is carried out while imposing a unit slope coefficient on inflation. That is, the factors are estimated from the real interest rate, $i_{i, t}-\pi_{i, t}$, which is consistent with the idea of the existence of a world real interest rate, see for example Lee (2002).

The principal components method is implemented as described in Section 5, but with the number of factors restricted to two, which ensures that the rank condition in Assumption 3 (iii) is fulfilled. As in the simulations, the defactored versions of the error correction tests of Westerlund (2007) are also considered. We focus on the results for Model 2 with an unrestricted constant, but include the results for Model 3 with both constant and trend for comparison.

The results reported in Table 8 suggest that there is strong evidence against the no cointegration null, even at the individual country level, which goes against the power argument of Westerlund (2008). Indeed, looking at the baseline specification with no trend, we end up rejecting the null for 13 out of the 20 countries when using the $\tau_{\tilde{\alpha}_{1 i}}$ test, and for 11 countries

\footnotetext{
${ }^{3}$ Although the panel tests of Westerlund (2008) are immune to the presence of common factors, his time series tests are not. This means that the two sets of results are not really comparable in the sense that the observed non-rejections at the individual country level could be due to the factors.

${ }^{4}$ One rationale for these factors is that they represent in part oil price shocks and other unanticipated changes in inflation.
} 
when using the $w_{\tilde{\delta}_{1 i}}$ test. Similarly, the pooled tests are way out in the critical region and lead to a safe rejection, even at the conservative $1 \%$ level. In other words, there is not much evidence against the Fisher effect. This conclusion is not altered by the inclusion of a linear trend.

In fact, the no cointegration null is rejected even when the factors are estimated with the slope on inflation fixed at unity. Specifically, although weaker at the individual level, the evidence at the overall panel level is still strong. Thus, we also have some evidence of the full Fisher effect.

To formally test for the presence of unit roots in the estimated factors, we follow the recommendation of Bai and $\mathrm{Ng}$ (2004) and use the augmented Dickey and Fuller (1979) test, ADF henceforth. ${ }^{5}$ The estimated first order AR coefficient for the two factors are 0.81 and 0.89 , indicating that there is considerable persistency in the factors. This evidence is reinforced by the associated ADF test values, -1.69 and -1.79 , respectively, which lead to an acceptance of the unit root null for both factors. Thus, if these factors are to be interpreted as emanating from the world real interest rate, then this rate must be non-stationary.

The lesson we draw from these results is that a failure to reject the null of no cointegration at the individual country level need not be taken as an indication of low power, as the possibility remains that it can be due to the presence of non-stationary common factors.

\subsection{The monetary exchange rate model}

In this section we take a closer look at the monetary exchange rate model, which postulates a strong link between the nominal exchange rate and a set of monetary fundamentals. The by far most scrutinized proposition being that the nominal exchange rate between the domestic and the foreign reference country, usually the United States, should cointegrate with the relative money supply and relative output of these countries.

However, as with the Fisher effect, despite its strong theoretical appeal, the empirical success of the monetary model has been rather limited, to say the least. Westerlund (2008), Mark and Sul (2001) and Rapach and Wohar (2004) for example argue that this is due to low power. They then proceed to show that the use of panel data leads to a much more favorable picture, with strong evidence of cointegration at the aggregate panel level. Therefore, since the countries appear to be cointegrated, the authors proceed to estimate the cointegration vector.

The problem is that since all variables are measured relative to the United States, this means that the common factors are there by construction. Furthermore, both money supply and output are generally believed to possess unit roots, even for the United States, such that

\footnotetext{
${ }^{5}$ The test allow for an intercept and the lag orders are determined using the Schwarz Bayesian information criterion.
} 
the common factors must be non-stationary as well. The potential consequences of unattended non-stationary factors on residual-based panel cointegration tests have been studied by Banerjee et al. (2004) and Gengenbach et al. (2006). The effects may lead to size distortions in small samples or even divergence in large samples. While Mark and Sul (2001) employ a block bootstrap to correct for some weak cross section dependence among the error term. It is not clear whether their test can correct for strong cross sectional dependence induced by non-stationary common factors. Rapach and Wohar (2004) only allow for cross section dependence in form of a common time effect.

In this section we revisit the results of Mark and Sul (2001) and Rapach and Wohar (2004). The data are taken directly from Mark and Sul (2001), and cover 18 countries between the first quarter of 1973 and the first quarter of 1997. Thus, in this application, $Y_{i, t}=e_{i, t}$ and

$$
X_{i, t}=\left(\begin{array}{c}
m_{t}^{*}-m_{i, t} \\
y_{t}^{*}-y_{i, t}
\end{array}\right)
$$

where $e_{i, t}, m_{i, t}$ and $y_{i, t}$ are the logarithm of the nominal exchange rate, money supply and real income for country $i$ at quarter $t$, respectively. Asterisks denote the United States.

The average-based tests are computed in the same ways as before, but now we consider two new versions of the factor-based tests. The first is based on using $m_{t}^{*}$ and $y_{t}^{*}$ as observed factors, which is very interesting in the sense that it provides an example of the scenario considered in Section 3.1. The second version is based on pre-specifying the cointegrating relationship as in Mark and Sul (2001). In particular, it is assumed that the relationship can be written as

$$
\beta^{\prime} Z_{i, t}=\left(\begin{array}{lll}
1, & -1, & 1
\end{array}\right)\left(\begin{array}{c}
Y_{i, t} \\
X_{i, t}
\end{array}\right)=e_{i, t}-\left(m_{t}^{*}-m_{i, t}\right)+\left(y_{t}^{*}-y_{i, t}\right),
$$

which imposes monetary neutrality and a unit negative income elasticity. ${ }^{6}$ Three factors are estimated from this relationship, which again ensures that Assumption 6 is satisfied. Once again we focus on Model 2 with an unrestricted constant as the deterministic component. For simplicity, in this section we drop the Westerlund (2007) tests.

The results are reported in Table 9. The first thing to note is that for the first 11 countries there is almost no evidence of cointegration at all, except possibly for Belgium, where we count four rejections at the $5 \%$ level. The pooled tests are generally much more significant, especially the Wald tests, displaying evidence of cointegration for all five panels. Just as before the results show almost no variation at all depending on whether the trend is included or not.

\footnotetext{
${ }^{6}$ In order to avoid the problem with nuisance parameter dependency discussed in Section 3.1, the cointegrating relationship is only pre-specified for the purpose of estimating the factors. In other words, although restricted in the factor estimation, in the implementation of the error correction tests the cointegrating relationship is still unrestricted.
} 
These two sets of results suggest that the evidence at the aggregate panel level could very well be due to only a few cointergrated countries. Indeed, a closer look at the different panel members reveals that the significance at the aggregate panel level is mainly due to three individually cointegrated countries, Italy, Spain and Korea. Although these differences could of course also be due to the relatively low power of the individual tests, they nevertheless show that one should take caution in interpreting test results at the aggregate panel level. Indeed, based on the results reported here it seems very hazardous, and borderline erroneous, to treat all five panels as cointegrated, and to proceed with the analysis as if all members are individually cointegrated.

When we compare the results from across the different tests, in agreement with our simulations, we see that the average-based Wald test leads to most rejections. As a final piece of evidence, Table 10 reports some summary statistics for the estimated factors. As in the Fisher application, we see that the estimated AR coefficients are very close to one, indicating the presence of unit roots, which is again supported by the ADF test results.

\section{Conclusions}

In this paper we consider the issue of testing for cointegration in a panel data model with non-stationary common factors. We begin by showing that the model admits to an ECM representation, a result that is then used for developing two new cointegration tests based on the significance of the error correction term.

It is shown that under the null of no error correction the asymptotic distributions of the tests are free of nuisance parameters, and that they only depend on the number of nonstationary variables in the system. However, the individual tests are not independent along the cross-sectional dimension, which makes pooling difficult. Nonetheless, the cross-sectional averages of these tests are shown to converge to well-defined distributions. These results hold regardless of whether the factors are treated as known or if they are estimated using the averages of the observed data. Some simulation evidence is also provided showing that the tests behave quite well in small samples.

A number of concluding remarks can be made. Firstly, the assumption of weak exogeneity of the regressors in the ECM is crucial for correct interpretation of the tests. This assumption is clearly a weakness in comparison to the residual-based test approach, in which the regressors can be fully endogenous by means of a non-parametric correction. However, it should be

pointed out that in principle there is nothing that precludes the use of a similar correction in the current setup. An alternative approach would be to pre-test the validity of the weak exogeneity assumption using panel extensions of the Lagrange multiplier tests proposed by Boswijk and Urbain (1997).

Secondly, the simulations show that the new tests can still be distorted in some cases 
when the factors are treated as unknown. One possibility towards this end would be to follow Palm et al. (2007), and to consider bootstrap versions of our tests, which are expected to have better size properties in small samples.

Finally, a crucial assumption is that of a single cointegrating vector under the alternative. This is obviously an important limitation of our tests that is shared with most existing residual-based tests. When the dimension of the cointegrating space is unknown it is probably best to analyze the data using system-based approaches, see for example Larsson et al. (2001). 


\section{References}

[1] Bai, J. and J. L. Carrion-i-Silvestre (2007). Testing for Panel Cointegration with Unobservable Dynamic Common Factors. Unpublished Manuscript.

[2] Bai, J., C. Kao and S. Ng. (2008). Panel Cointegration with Global Stochastic Trends. Unpublished Manuscript.

[3] Bai, J., and S. Ng. (2002). Determining the Number of Factors in Approximate Factor Models. Econometrica 70, 191-221.

[4] Bai, J., and S. Ng. (2004). A PANIC Attack on Unit Roots and Cointegration. Econometrica 72, 1127-1177.

[5] Banerjee, A. and J. L. Carrion-i-Silvestre (2007). Cointegration in Panel Data with Breaks and Cross-Section Dependence. Economics Working Papers ECO2006/5, European University Institute.

[6] Banerjee, A., M. Marcellino, and C. Osbat (2004). Some Cautions on the Use of Panel Methods for Integrated Time Series of Macroeconomic Data. Econometrics Journal 7, $322-340$.

[7] Boswijk, P. H. (1994). Testing for an Unstable Root in Conditional and Structural Error Correction Models. Journal of Econometrics 63, 37-60.

[8] Boswijk, P. H., and J.-P. Urbain (1997). Lagrange Multiplier Tests for Weak Exogeneity: A Synthesis. Econometric Reviews 16, 21-38.

[9] Breitung, J., and M. H. Pesaran (2008). Unit Roots and Cointegration in Panels. In Matyas, L., and P. Sevestre (Eds.) The Econometrics of Panel Data: Fundamentals and Recent Developments in Theory and Practice, 279-322. Kluwer Academic Publishers, Boston.

[10] Cappuccio, N., and D. Lubian (1996). Triangular Representation and Error Correction Mechanism in Cointegrated Systems. Oxford Bulletin of Economics and Statistics 58, 409-415.

[11] Engle, R. F., and C. W. J. Granger (1987). Co-Integration and Error Correction: Representation, Estimation and Testing. Econometrica 55, 251-276.

[12] Gengenbach, C., F. C. Palm and J.-P. Urbain (2006). Panel Cointegration Testing in the Presence of Common Factors. Oxford Bulletin of Economics and Statistics 68, pp. 683-719. 
[13] Gregoir, S. (2005). Representaion and Statistical Analysis of Weakly Linearly Exchangable Dynamic Panels. Working Paper, CREST.

[14] Im, K. S., M. Peseran and Y. Shin (2003). Testing for Unit Roots in Heterogeneous Panels. Journal of Econometrics 115, 53-74.

[15] Larsson, R., J. Lyhagen and M. Lötgren (2001). Likelihood-Based Cointegration Test in Heterogeneous Panels. Econometrics Journal 4, 109-142.

[16] Lee, J. E. (2002). On the Characterisation of the World Real Interest Rate. World Economy 25, 247-255.

[17] Mark, N. C., and D. Sul (2001). Nominal Exchange Rates and Monetary Fundamentals: Evidence from a Small Post-Bretton Woods Panel. Journal of International Economics 53, pp. 29-52.

[18] Ng, S., and P. Perron (1995). Unit Root Test in ARMA Models with Data-Dependent Methods for the Selection of the Truncation Lag. Journal of the American Statistical Association 90, 268-281.

[19] Palm, F., S. Smeekes and J.-P. Urbain (2007). A Sieve Bootstrap Test for Cointegration in a Conditional Error Correction Model. METEOR Research Memorandum 07054, Maastricht University.

[20] Park, J. Y., and P. C. B. Phillips (1988). Statistical Inference in Regressions with Integrated Processes: Part 1. Econometric Theory 4, 468-497.

[21] Pedroni, P. (1999). Critical Values for Cointegration Tests in Heterogeneous Panels with Multiple Regressors. Oxford Bulletin of Economics and Statistics 61, 653-670.

[22] Pedroni, P. (2004). Panel Cointegration: Asymptotic and Finite Sample Properties of Pooled Time Series Tests with an Application to the PPP Hypothesis. Econometric Theory 20, 597-625.

[23] Pesaran, M. H. (2007). A Simple Panel Unit Root Test in the Presence of Cross Section Dependence. Journal of Applied Econometrics 22, 265-312.

[24] Pesavento, E. (2004). Analytical Evaluation of the Power of Tests for the Absence of Cointegration. Journal of Econometrics 122, 349-384.

[25] Phillips, P. C. B., and H. R. Moon (1999). Linear Regression Limit Theory for Nonstationary Panel Data. Econometrica 67, 1057-1111. 
[26] Rapach, D. E., and M. E. Wohar (2004). Testing the Monetary Model of Exchange Rate Determination: A Closer Look at Panels. Journal of International Money and Finance 23, 867-895.

[27] Saikkonen, P. (1991). Asymptotically Efficient Estimation of Cointegration Regressions. Econometric Theory 7, 1-21.

[28] Urbain, J.-P. (1992). On Weak Exogeneity in Error Correction Models, Oxford Bulletin of Economics and Statistics 54, 187-206.

[29] Westerlund, J. (2007). Testing for Error Correction in Panel Data. Oxford Bulletin of Economics and Statistics 69, 709-748.

[30] Westerlund, J. (2008). Panel Cointegration Tests of the Fisher Effect. Journal of Applied Econometrics 23, 193-233.

[31] Westerlund, J., and R. Larsson (2008). A Note on the Pooling of Individual PANIC Unit Root Tests. Unpublished manuscript.

[32] Zivot, E. (2000). The Power of Single Equation Tests for Cointegration when the Cointegration Vector is Prespecified. Econometric Theory 16, 407-439. 


\section{A Appendix}

\section{A.1 Proof of Theorem 1.}

Consider (a). From (3) and (4) we have $\Delta F_{t}-\pi_{3}^{\prime} g_{t}=\Psi(L) \eta_{t}$. Substituting for $\Delta F_{t}$ in (2) and using (4) we obtain

$$
\Delta X_{i, t}-\pi_{2 i}^{*}{ }^{\prime} g_{t}=\Gamma_{21 i}(L) \varepsilon_{1 i, t}+\Gamma_{22 i}(L) \varepsilon_{2 i, t}+\lambda_{2 i}^{\prime} \Psi(L) \eta_{t} .
$$

Taking first differences of (1) and substituting for $\Delta F_{t}$ and $\Delta X_{i, t}$ from (A1) we obtain the following MA representation

$$
\begin{aligned}
\Delta Y_{i, t}-\pi_{1 i}^{* \prime} g_{t}= & \left((1-L) \Gamma_{11 i} b_{i}^{\prime} \Gamma_{21 i}(L)\right) \varepsilon_{1 i, t} \\
& +\left((1-L) \Gamma_{21 i}+b_{i}^{\prime} \Gamma_{22 i}(L)\right) \varepsilon_{2 i, t}+\left(\lambda_{1 i}^{\prime}+b_{i}^{\prime} \lambda_{2 i}^{\prime}\right) \Psi(L) \eta_{t} .
\end{aligned}
$$

Combining the results above we find

$$
C_{i}(L)=\left(\begin{array}{ccc}
(1-L) \Gamma_{11 i}(L)+b_{i}^{\prime} \Gamma_{21 i}(L) & (1-L) \Gamma_{12 i}(L)+b_{i}^{\prime} \Gamma_{22 i}(L) & \left(\lambda_{1 i}^{\prime}+b_{i}^{\prime} \lambda_{2 i}^{\prime}\right) \Psi(L) \\
\Gamma_{21 i}(L) & \Gamma_{22 i}(L) & \lambda_{2 i}^{\prime} \Psi(L) \\
0 & 0 & \Psi(L)
\end{array}\right) .
$$

Furthermore,

$$
C_{i}(1)=\left(\begin{array}{cc}
b_{i}^{\prime} & \left(\lambda_{1 i}^{\prime}+b_{i}^{\prime} \lambda_{2 i}^{\prime}\right) \\
I_{m} & \lambda_{2 i}^{\prime} \\
0 & I_{k}
\end{array}\right)\left(\begin{array}{ccc}
\Gamma_{21 i}(1) & \Gamma_{22 i}(1) & 0 \\
0 & 0 & \Psi(1)
\end{array}\right)
$$

such that $C_{i}(1)$ has rank $m+k$. This establishes part (a) of the theorem.

Next, consider (b). Partition $C_{i}(L)$ such that the diagonal blocks $C_{11 i}(L)$ and $C_{22 i}(L)$ are of dimension $r \times r$ and $(m+k) \times(m+k)$, respectively. Since

$$
C_{22 i}(L)=\left(\begin{array}{cc}
\Gamma_{22 i}(L) & \lambda_{2 i}^{\prime} \Psi(L) \\
0 & \Psi(L)
\end{array}\right)
$$

is invertible, we can decompose $C_{i}(L)$ as

$$
C_{i}(L)=\left(\begin{array}{cc}
I_{r} & C_{12 i}(L) C_{22 i}(L)^{-1} \\
0 & I_{(m+k)}
\end{array}\right)\left(\begin{array}{cc}
C_{11 \cdot 2 i}(L) & 0 \\
C_{21 i}(L) & C_{22 i}(L)
\end{array}\right) .
$$

As $C_{11 \cdot 2 i}=(1-L) \Gamma_{11 \cdot 2 i}(L)$ we can further write

$$
\begin{aligned}
C_{i}(L) & =\left(\begin{array}{cc}
I_{r} & C_{12 i}(L) C_{22 i}(L)^{-1} \\
0 & I_{(m+k)}
\end{array}\right)\left(\begin{array}{cc}
(1-L) I_{r} & 0 \\
0 & I_{(m+k)}
\end{array}\right)\left(\begin{array}{cc}
\Gamma_{11 \cdot 2 i}(L) & 0 \\
C_{21 i}(L) & C_{22 i}(L)
\end{array}\right) \\
& =U_{i}(L)^{-1} M(L) V_{i}(L)^{-1},
\end{aligned}
$$

where the lag polynomials

$$
V_{i}(L)=\left(\begin{array}{ccc}
\Gamma_{11 \cdot 2 i}(L)^{-1} & 0 & 0 \\
-\Gamma_{22 i}(L)^{-1} \Gamma_{21 i}(L) \Gamma_{11 \cdot 2 i}(L)^{-1} & \Gamma_{22 i}(L)^{-1} & -\Gamma_{22 i}(L)^{-1} \lambda_{2 i}^{\prime} \\
0 & 0 & \Psi(L)^{-1}
\end{array}\right)
$$


and

$$
U_{i}(L)=\left(\begin{array}{ccc}
I_{r} & -\left((1-L) \Gamma_{12 i}(L) \Gamma_{22 i}(L)^{-1}+b_{i}^{\prime}\right) & (1-L) \Gamma_{12 i}(L) \Gamma_{22 i}(L)^{-1} \lambda_{2 i}^{\prime}-\lambda_{1 i}^{\prime} \\
0 & I_{m} & 0 \\
0 & 0 & I_{k}
\end{array}\right)
$$

are invertible.

Substituting (A3) for $C_{i}(L)$ in the MA representation of $\Delta Z_{i, t}^{+}$and pre-multiplying by $U_{i}(L)$ and $\bar{M}(L)$, where

$$
\bar{M}(L)=\left(\begin{array}{cc}
I_{r} & 0 \\
0 & (1-L) I_{(m+k)}
\end{array}\right)
$$

we obtain

$$
\bar{M}(L) U_{i}(L)(1-L)\left(Z_{i, t}^{+}-\left(\pi_{i}^{*}\right)^{\prime} g_{t}\right)=(1-L) V_{i}(L)^{-1} \varepsilon_{i, t}^{+} .
$$

Eliminating $(1-L)$ from both sides and pre-multiplying by $V_{i}(L)$ yields the following possibly infinite $\mathrm{AR}$ representation for $Z_{i, t}^{+}$

$$
V_{i}(L) \bar{M}(L) U_{i}(L)\left(Z_{i, t}^{+}-\left(\pi_{i}^{*}\right)^{\prime} g_{t}\right)=\varepsilon_{i, t}^{+} .
$$

Using that $\Gamma_{11 \cdot 2 i}(L)^{-1}=\left|\Gamma_{11 \cdot 2 i}(L)\right|^{-1} \operatorname{adj}\left(\Gamma_{11 \cdot 2 i}(L)\right), \Gamma_{22 i}(L)^{-1}=\left|\Gamma_{22 i}(L)\right|^{-1} \operatorname{adj}\left(\Gamma_{22 i}(L)\right)$, $\Psi(L)^{-1}=|\Psi(L)|^{-1} \operatorname{adj}(\Psi(L))$ and $\left|\Gamma_{i}^{+}(L)\right|=\left|\Gamma_{11 \cdot 2 i}(L)\right|\left|\Gamma_{22 i}(L)\right||\Psi(L)|$, we can recover both the scalar lag polynomial $c_{i}(L)=\left|\Gamma_{i}^{+}(L)\right|$ and the lag polynomial matrix $A_{i}(L)$ given in the theorem. This establishes part (b).

Consider (c). Direct computation of $A_{i}(1)$ yields

$$
A_{i}(1)=\left(\begin{array}{c}
|\Psi(1)|\left|\Gamma_{22 i}(1)\right| \operatorname{adj}\left(\Gamma_{11 \cdot 2 i}(1)\right) \\
-|\Psi(1)| \operatorname{adj}\left(\Gamma_{22 i}(1)\right) \Gamma_{21 i}(1) \operatorname{adj}\left(\Gamma_{11 \cdot 2 i}(1)\right) \\
0
\end{array}\right)\left(\begin{array}{lll}
I_{r} & -b_{i}^{\prime} & -\lambda_{1 i}^{\prime}
\end{array}\right)=\alpha_{i}^{*} \beta_{i}^{\prime} .
$$

Since

$$
C_{i}(1)=\left(\begin{array}{cc}
b_{i}^{\prime} & \left(\lambda_{1 i}^{\prime}+b_{i}^{\prime} \lambda_{2 i}^{\prime}\right) \\
I_{m} & \lambda_{2 i}^{\prime} \\
0 & I_{k}
\end{array}\right)\left(\begin{array}{ccc}
\Gamma_{21 i}(1) & \Gamma_{22 i}(1) & 0 \\
0 & 0 & \Psi(1)
\end{array}\right)=\tilde{\beta}_{i}\left(\tilde{\alpha}_{i}^{*}\right)^{\prime},
$$

where $\tilde{\alpha}_{i}^{*}$ and $\tilde{\beta}_{i}$ denote the matrices orthogonal to $\alpha_{i}^{*}$ and $\beta_{i}$, respectively. It follows that $\beta_{i}^{\prime} C_{i}(1)=0$ and $C_{i}(1) \alpha_{i}=0$, and so the proof of (c) is complete.

Parts (d) and (e) follow by manipulating of the lag polynomial matrix $A_{i}(L)$ and rearranging terms, as in Engle and Granger (1987).

\section{A.2 Proof of Theorem 2.}

Before we come to the proof of the theorem we need some preliminary results, which are summarized in Lemma 1.

Lemma 1. Under $H_{0 i}$ and Assumptions 1, 2, 4 and 5, as $T \rightarrow \infty$ 
(a) $T^{-\frac{1}{2}} Z_{i, t-1}^{+} \stackrel{w}{\longrightarrow} \Omega_{i}^{\frac{1}{2}} W_{i}$,

(b) $T^{-2} \sum_{t=2}^{T} Q_{W} Z_{i, t-1}^{+}\left(Q_{W} Z_{i, t-1}^{+}\right)^{\prime} \stackrel{w}{\longrightarrow} \Omega_{i}^{\frac{1}{2}} \int W_{i} W_{i}^{\prime}\left(\Omega_{i}^{\frac{1}{2}}\right)^{\prime}$,

(c) $T^{-1} \sum_{t=2}^{T} Q_{W} Z_{i, t-1}^{+} Q_{W} \varepsilon_{1 \cdot 2 i, t} \stackrel{w}{\longrightarrow} \sigma_{i} \Omega_{i}^{\frac{1}{2}} p_{i}\left(W_{i}\right)$,

where

$$
\begin{aligned}
\Omega_{i}^{\frac{1}{2}} & =\left(\begin{array}{cc}
\sigma_{i} \tilde{B}_{11 \cdot 2 i}^{-1} & \tilde{B}_{11 \cdot 2 i}^{-1} M_{1 i} M_{2 i} \\
\sigma_{i} \tilde{B}_{11 \cdot 2 i}^{-1} \rho_{i} & \left(M_{2 i}+\tilde{B}_{11 \cdot 2 i}^{-1} \rho_{i} M_{1 i} M_{2 i}\right)
\end{array}\right) \\
& =\left(\begin{array}{cc}
\omega_{11 i} & \Omega_{12 i} \\
\Omega_{21 i} & \Omega_{22 i}
\end{array}\right),
\end{aligned}
$$

where $\tilde{B}_{j j i}(L)=I-B_{j j i}(L) L, B_{j l i}=B_{j l i}(1), \tilde{B}_{j l i}=\tilde{B}_{j l i}(1), \tilde{B}_{11 \cdot 2 i}^{-1}=\left(\tilde{B}_{11 i}-B_{12 i} \tilde{B}_{22 i}^{-1} B_{21 i}\right)^{-1}$, $\rho_{i}^{\prime}=\left(\begin{array}{ll}B_{21 i}^{\prime}\left(\tilde{B}_{22 i}^{-1}\right)^{\prime} & 0\end{array}\right), M_{1 i}=\left(\begin{array}{cc}B_{12 i} & B_{13 i}\end{array}\right)$ and

$$
M_{2 i}=\left(\begin{array}{cc}
\tilde{B}_{22 i}^{-1} \Sigma_{22 i}^{\frac{1}{2}} & \tilde{B}_{22 i}^{-1}\left(B_{23 i} \tilde{B}_{33 i}^{-1}+\lambda_{2 i}^{\prime}\right) \\
0 & \tilde{B}_{33 i}^{-1}
\end{array}\right) .
$$

Note that $\Omega_{21 i}=\omega_{11 i} \rho_{i}$ and define for future use $\omega_{11 \cdot 2 i}=\omega_{11 i}-\omega_{11 i} \Omega_{12} \Omega_{22 i}^{-1} \rho_{i}$.

\section{Proof of Lemma 1.}

Consider (a). Note that under $H_{0 i}$,

$$
\left(\begin{array}{ccc}
\tilde{B}_{11 i}(1) & -B_{12 i}(1) & -B_{13 i}(1) \\
-B_{21 i}(1) L & \tilde{B}_{22 i}(1) & -B_{23 i}(1) L \\
0 & 0 & \tilde{B}_{33 i}(1)
\end{array}\right) \Delta Z_{i, t}^{+}=\left(\begin{array}{c}
\varepsilon_{1 \cdot 2 i, t} \\
\varepsilon_{2 i, t}^{*} \\
\eta_{t}
\end{array}\right),
$$

such that

$$
\begin{aligned}
& Z_{i, t}^{+}=\left(\begin{array}{ccc}
\tilde{B}_{11 i}(1) & -B_{12 i}(1) & -B_{13 i}(1) \\
-B_{21 i}(1) & \tilde{B}_{22 i}(1) & -B_{23 i}(1) \\
0 & 0 & \tilde{B}_{33 i}(1)
\end{array}\right)^{-1}\left(\sum_{s=1}^{t}\left(\begin{array}{c}
\varepsilon_{1 \cdot 2 i, t} \\
\varepsilon_{2 i, t}^{*} \\
\eta_{t}
\end{array}\right)\right. \\
& \left.+\left(\begin{array}{ccc}
-\tilde{B}_{11 i}^{+}(L) & B_{12 i}^{+}(L) & B_{13 i}^{+}(L) \\
B_{21 i}^{+}(L) L & -\tilde{B}_{22 i}^{+}(L) & B_{23 i}^{+}(L) L \\
0 & 0 & -\tilde{B}_{33 i}^{+}(L)
\end{array}\right) \Delta Z_{i, t}^{+}\right),
\end{aligned}
$$

where $B_{j l i}^{+}(L)$ and $\tilde{B}_{j l i}^{+}(L)$ are obtained from the Beveridge-Nelson decompositions of $B_{j l i}(L)$ and $\tilde{B}_{j l i}(L)$ as $B_{j l i}(L)=B_{j l i}(1)+B_{j l i}^{+}(L)(1-L)$ and $\tilde{B}_{j l i}(L)=\tilde{B}_{j l i}(1)+\tilde{B}_{j l i}^{+}(L)(1-L)$, respectively. 
Substituting $\varepsilon_{2 i, t}^{*}=\varepsilon_{2 i, t}+\lambda_{2 i}^{\prime} \eta_{t}$ into (A4) we obtain

$$
\begin{aligned}
T^{-\frac{1}{2}} Z_{i, t}^{+} & =\left(\begin{array}{ccc}
\tilde{B}_{11 i}(1) & -B_{12 i}(1) & -B_{13 i}(1) \\
-B_{21 i}(1) & \tilde{B}_{22 i}(1) & -B_{23 i}(1) \\
0 & 0 & \tilde{B}_{33 i}(1)
\end{array}\right)^{-1}\left(\begin{array}{ccc}
\sigma_{i} & 0 & 0 \\
0 & \Sigma_{22 i}^{\frac{1}{2}} & \lambda_{2 i}^{\prime} \\
0 & 0 & I_{k}
\end{array}\right) \\
& \times T^{-\frac{1}{2}} \sum_{s=1}^{t}\left(\begin{array}{c}
\sigma_{i}^{-1} \varepsilon_{1 \cdot 2 i, t} \\
\Sigma_{22 i}^{-\frac{1}{2}} \varepsilon_{2 i, t} \\
\eta_{t}
\end{array}\right)+o_{p}(1),
\end{aligned}
$$

such that

$$
T^{-\frac{1}{2}} Z_{i, t}^{+} \stackrel{w}{\longrightarrow} \Omega_{i}^{\frac{1}{2}} W_{i}
$$

as $T \rightarrow \infty$, proving (a).

Now, by using the rules for projections, $\sum_{t=2}^{T} Q_{W} Z_{i, t-1}^{+}\left(Q_{W} Z_{i, t-1}^{+}\right)^{\prime}$ can be written as

$$
\begin{aligned}
\sum_{t=2}^{T} Q_{W} Z_{i, t-1}^{+}\left(Q_{W} Z_{i, t-1}^{+}\right)^{\prime} & =\sum_{t=2}^{T} Z_{i, t-1}^{+}\left(Z_{i, t-1}^{+}\right)^{\prime} \\
& -\sum_{t=2}^{T} Z_{i, t-1}^{+} W_{i, t}^{\prime}\left(\sum_{t=2}^{T} W_{i, t} W_{i, t}^{\prime}\right)^{-1} \sum_{t=2}^{T} W_{i, t}\left(Z_{i, t-1}^{+}\right)^{\prime} .
\end{aligned}
$$

By Lemma 2.1 of Park and Phillips (1989), $\sum_{t=2}^{T} Z_{i, t-1}^{+} W_{i, t}^{\prime}=O_{p}(T), \sum_{t=2}^{T} Z_{i, t-1}^{+}\left(Z_{i, t-1}^{+}\right)^{\prime}=$ $O_{p}\left(T^{2}\right)$ and $\sum_{t=2}^{T} W_{i, t} W_{i, t}^{\prime}=O_{p}(T)$ such that (A6) reduces to

$$
\begin{aligned}
T^{-2} \sum_{t=2}^{T} Q_{W} Z_{i, t-1}^{+}\left(Q_{W} Z_{i, t-1}^{+}\right)^{\prime} & =T^{-2} \sum_{t=2}^{T} Z_{i, t-1}^{+}\left(Z_{i, t-1}^{+}\right)^{\prime}+T^{-2} O_{p}(T) O_{p}\left(T^{-1}\right) O_{p}(T) \\
& =T^{-2} \sum_{t=2}^{T} Z_{i, t-1}^{+}\left(Z_{i, t-1}^{+}\right)^{\prime}+O_{p}\left(T^{-1}\right)
\end{aligned}
$$

where we can make use of (a) to show that as $T \rightarrow \infty$

$$
T^{-2} \sum_{t=2}^{T} Z_{i, t-1}^{+}\left(Z_{i, t-1}^{+}\right)^{\prime} \stackrel{w}{\longrightarrow} \Omega_{i}^{\frac{1}{2}} \int W_{i} W_{i}^{\prime}\left(\Omega_{i}^{\frac{1}{2}}\right)^{\prime}
$$

This proves (b).

Finally, consider (c). By definition,

$$
\sum_{t=2}^{T} Q_{W} Z_{i, t-1}^{+}\left(Q_{W} \varepsilon_{1 \cdot 2 i, t}\right)=\sum_{t=2}^{T} Z_{i, t-1}^{+} \varepsilon_{1 \cdot 2 i, t}-\sum_{t=2}^{T} Z_{i, t-1}^{+} W_{i, t}^{\prime}\left(\sum_{t=2}^{T} W_{i, t} W_{i, t}^{\prime}\right)^{-1} \sum_{t=2}^{T} W_{i, t} \varepsilon_{1 \cdot 2 i, t}
$$

where $\sum_{t=2}^{T} W_{i, t} \varepsilon_{1 \cdot 2 i, t}=O_{p}(\sqrt{T})$. Thus, by using the same arguments as above,

$$
T^{-1} \sum_{t=2}^{T} Q_{W} Z_{i, t-1}^{+}\left(Q_{W} \varepsilon_{1 \cdot 2 i, t}\right)=T^{-1} \sum_{t=2}^{T} Z_{i, t-1}^{+} \varepsilon_{1 \cdot 2 i, t}+O_{p}\left(T^{-\frac{1}{2}}\right),
$$


where the limit of the first term on the right-hand side is given by

$$
T^{-1} \sum_{t=2}^{T} Z_{i, t-1}^{+} \varepsilon_{1 \cdot 2 i, t} \stackrel{w}{\longrightarrow} \sigma_{i} \Omega_{i}^{\frac{1}{2}} \int W_{i} d W_{1 i}=\sigma_{i} \Omega_{i}^{\frac{1}{2}} p_{i}\left(W_{i}\right) .
$$

This establishes (c), and hence the proof of Lemma 1 is complete.

Now, since under the null hypothesis,

$$
\Delta\left(Q_{W} Y_{i, t}\right)=\delta_{1 i}^{\prime}\left(Q_{W} Z_{i, t-1}^{+}\right)+Q_{W} \varepsilon_{1 \cdot 2 i, t}=Q_{W} \varepsilon_{1 \cdot 2 i, t}
$$

we have

$$
\hat{\delta}_{1 i}=\left(\sum_{t=2}^{T} Q_{W} Z_{i, t-1}^{+}\left(Q_{W} Z_{i, t-1}^{+}\right)^{\prime}\right)^{-1} \sum_{t=2}^{T} Q_{W} Z_{i, t-1}^{+}\left(Q_{W} \varepsilon_{1 \cdot 2 i, t}\right) .
$$

From Lemma 1 (b) and (c) we have that

$$
T \hat{\delta}_{1 i} \stackrel{w}{\longrightarrow} \sigma_{i}\left(\Omega_{i}^{\frac{1}{2}} \int W_{i} W_{i}^{\prime}\left(\Omega_{i}^{\frac{1}{2}}\right)^{\prime}\right)^{-1} \Omega_{i}^{\frac{1}{2}} \int W_{i} d W_{1 i}=\sigma_{i}\left(\Omega_{i}^{-\frac{1}{2}}\right)^{\prime} P_{i}\left(W_{i}\right) .
$$

Similarly, under the null the Wald statistic is given by

$$
w_{\hat{\delta}_{1 i}}=\hat{\sigma}_{i}^{-2} \sum_{t=2}^{T} Q_{W} \varepsilon_{1 \cdot 2 i, t}\left(Q_{W} Z_{i, t}^{+}\right)^{\prime}\left(\sum_{t=2}^{T} Q_{W} Z_{i, t-1}^{+}\left(Q_{W} Z_{i, t-1}^{+}\right)^{\prime}\right)^{-1} \sum_{t=2}^{T} Q_{W} Z_{i, t-1}^{+}\left(Q_{W} \varepsilon_{1 \cdot 2 i, t}\right) .
$$

Consider $\hat{\sigma}_{i}^{2}=T^{-1} \sum_{t=2}^{T}\left(\Delta\left(Q_{W} Y_{i, t}\right)-\hat{\delta}_{1 i}^{\prime}\left(Q_{W} Z_{i, t-1}^{+}\right)\right)^{2}$. By making use of Lemma 1 , and the fact that under the null, $\Delta\left(Q_{W} Y_{i, t}\right)=Q_{W} \varepsilon_{1 \cdot 2 i, t}$, we get

$$
\begin{aligned}
\hat{\sigma}_{i}^{2} & =T^{-1} \sum_{t=2}^{T}\left(\Delta\left(Q_{W} Y_{i, t}-\hat{\delta}_{1 i}\left(Q_{W} Z_{i, t-1}^{+}\right)\right)^{2}\right. \\
& =T^{-1} \sum_{t=2}^{T}\left(Q_{W} \varepsilon_{1 \cdot 2 i, t}\right)^{2}-2 \hat{\delta}_{1 i}^{\prime} T^{-1} \sum_{t=2}^{T} Q_{W} Z_{i, t-1}^{+}\left(Q_{W} \varepsilon_{1 \cdot 2 i, t}\right) \\
& +\hat{\delta}_{1 i}^{\prime} T^{-1} \sum_{t=2}^{T} Q_{W} Z_{i, t-1}^{+}\left(Q_{W} Z_{i, t-1}^{+}\right)^{\prime} \hat{\delta}_{1 i} \\
& =T^{-1} \sum_{t=2}^{T}\left(Q_{W} \varepsilon_{1 \cdot 2 i, t}\right)^{2}+O_{p}\left(T^{-1}\right) O_{p}(1)+T^{-1} O_{p}\left(T^{-1}\right) O_{p}\left(T^{2}\right) O_{p}\left(T^{-1}\right) \\
& =T^{-1} \sum_{t=2}^{T}\left(Q_{W} \varepsilon_{1 \cdot 2 i, t}\right)^{2}+O_{p}\left(T^{-1}\right) .
\end{aligned}
$$

As for the first term on the right-hand side, we have

$$
\begin{aligned}
\sum_{t=2}^{T}\left(Q_{W} \varepsilon_{1 \cdot 2 i, t}\right)^{2} & =\sum_{t=2}^{T} \varepsilon_{1 \cdot 2 i, t}^{2}-\sum_{t=2}^{T} \varepsilon_{1 \cdot 2 i, t} W_{i, t}^{\prime}\left(\sum_{t=2}^{T} W_{i, t} W_{i, t}^{\prime}\right)^{-1} \sum_{t=2}^{T} W_{i, t} \varepsilon_{1 \cdot 2 i, t} \\
& =\sum_{t=2}^{T} \varepsilon_{1 \cdot 2 i, t}^{2}+O_{p}(\sqrt{T}) O_{p}\left(T^{-1}\right) O_{p}(\sqrt{T}) .
\end{aligned}
$$


Hence, by inserting this into (A9), and then taking the limit as $T \rightarrow \infty$, we obtain

$$
\hat{\sigma}_{i}^{2}=T^{-1} \sum_{t=2}^{T} \varepsilon_{1 \cdot 2 i, t}^{2}+O_{p}\left(T^{-1}\right) \stackrel{p}{\longrightarrow} \sigma_{i}^{2} .
$$

Combining this result with Lemma 1 (b) and (c) we get the following limit as $T \rightarrow \infty$

$$
\begin{aligned}
w_{\hat{\delta}_{1 i}} & \stackrel{w}{\longrightarrow} \sigma_{i}^{-2} \sigma_{i} \int d W_{1 i} W_{i}^{\prime}\left(\Omega_{i}^{\frac{1}{2}}\right)^{\prime}\left(\Omega_{i}^{\frac{1}{2}} \int W_{i} W_{i}^{\prime}\left(\Omega_{i}^{\frac{1}{2}}\right)^{\prime}\right)^{-1} \sigma_{i} \Omega_{i}^{\frac{1}{2}} \int W_{i} d W_{1 i} \\
& =p_{i}\left(W_{i}\right)^{\prime} P_{i}\left(W_{i}\right),
\end{aligned}
$$

which establishes part (a) of the theorem.

Consider (b). Under the null hypothesis, $\Delta\left(Q_{S} Y_{i, t}\right)=Q_{S} \varepsilon_{1 \cdot 2 i, t}$. By using this result, (A8) and the rules for partitioned regressions, we obtain as $T \rightarrow \infty$

$$
\begin{aligned}
T \hat{\alpha}_{1 i} & =\left(T^{-2} \sum_{t=2}^{T}\left(Q_{S} Y_{i, t-1}\right)^{2}\right)^{-1} T^{-1} \sum_{t=2}^{T} Q_{S} Y_{i, t-1}\left(Q_{S} \varepsilon_{1 \cdot 2 i, t}\right) \\
& \stackrel{w}{\longrightarrow} \sigma_{i} \omega_{11 \cdot 2 i}^{-1} P_{i}\left(U_{i}\right)+\omega_{11 \cdot 2 i}^{-1} \omega_{11 i} \rho_{i}^{\prime}\left(\Omega_{22 i}^{\prime}\right)^{-1} P_{i}\left(W_{2 \cdot i}\right)\left(P_{i}\left(U_{i}\right)-1\right)=d_{i} .
\end{aligned}
$$

Next, consider

$$
\operatorname{var}\left(\hat{\alpha}_{1 i}\right)=\hat{\sigma}_{i}^{2}\left(T^{-2} \sum_{t=2}^{T}\left(Q_{S} Y_{i, t-1}\right)^{2}\right)^{-1} .
$$

We have already shown that $\hat{\sigma}_{i}^{2} \stackrel{p}{\longrightarrow} \sigma_{i}^{2}$ as $T \rightarrow \infty$. From this result and arguments similar to those used in the proof of Lemma 1 we obtain as $T \rightarrow \infty$

$$
\begin{aligned}
T^{2} \operatorname{var}\left(\hat{\alpha}_{1 i}\right) & \stackrel{w}{\longrightarrow} \sigma_{i}^{2} \omega_{11 \cdot 2 i}^{-2} V\left(U_{i}\right) \\
& +\sigma_{i}^{2} \omega_{11 \cdot 2 i}^{-2} \omega_{11 i} V\left(U_{i}\right)\left(\rho_{i}^{\prime}\left(\Omega_{22 i}^{\prime}\right)^{-1} P_{i}\left(W_{2 \cdot i}\right)+V\left(W_{2 \cdot i}\right) p_{i}\left(W_{2 \cdot i}\right)^{\prime} \Omega_{22 i}^{-1} \rho_{i}\right) \\
& +\sigma_{i}^{2} \omega_{11 \cdot 2 i}^{-2} \omega_{11 i}^{2} \rho_{i}^{\prime}\left(\Omega_{22 i}^{\prime}\right)^{-1} \\
& \times\left(V\left(W_{2 \cdot i}\right)+P_{i}\left(W_{2 \cdot i}\right) V\left(U_{i}\right) V\left(W_{2 \cdot i}\right) p_{i}\left(W_{2 \cdot i}\right)^{\prime}\right) \Omega_{22 i}^{-1} \rho_{i}=D_{i} .
\end{aligned}
$$

The proof is completed by noting that

$$
D_{i, \tau}^{\Omega}=\lim _{T \rightarrow \infty} \frac{T \hat{\alpha}_{1 i}}{\sqrt{T^{2} \operatorname{var}\left(\hat{\alpha}_{1 i}\right)}}=\frac{d_{i}}{\sqrt{D_{i}}} .
$$

\section{A.2.1 Proof of Corollary 1}

If $X_{i, t}$ is strongly exogenous, $B_{21 i}=0$ such that $\rho_{i}=0$. Thus, (A14) simplifies to

$$
D_{i, \tau}=\frac{d_{i}}{\sqrt{D_{i}}}=\frac{\tilde{B}_{11 i} P_{i}\left(U_{i}\right)}{\sqrt{\tilde{B}_{11 i}^{2} V\left(U_{i}\right)}}=\frac{P_{i}\left(U_{i}\right)}{\sqrt{V\left(U_{i}\right)}} .
$$

This completes the proof. 


\section{A.3 Proof of Theorem 3}

We begin with the following lemma.

Lemma 2. Under $H_{0 i}$ and Assumptions 1 to 6 , as $N, T \rightarrow \infty$

(a) $T^{-\frac{1}{2}} \bar{Z}_{t} \stackrel{w}{\longrightarrow} M_{3} W_{3}$,

(b) $T^{-\frac{1}{2}} \tilde{Z}_{i, t-1}^{+} \stackrel{w}{\longrightarrow} \tilde{\Omega}_{i}^{\frac{1}{2}} W_{i}$,

(c) $T^{-2} \sum_{t=2}^{T} Q_{\tilde{W}^{\prime}} \tilde{Z}_{i, t}^{+}\left(Q_{\tilde{W}} \tilde{Z}_{i, t}^{+}\right)^{\prime} \stackrel{w}{\longrightarrow} \tilde{\Omega}_{i}^{\frac{1}{2}}\left(\int W_{i} W_{i}^{\prime}\right)\left(\tilde{\Omega}_{i}^{\frac{1}{2}}\right)^{\prime}$,

(d) $T^{-1} \sum_{t=2}^{T} Q_{\tilde{W}} \tilde{Z}_{i, t-1}^{+}\left(Q_{\tilde{W}} \tilde{\varepsilon}_{1 \cdot 2 i, t}\right) \stackrel{w}{\longrightarrow} \sigma_{i} \tilde{\Omega}_{i}^{\frac{1}{2}} p_{i}\left(W_{i}\right)$,

where $M_{3}=\lim _{N \rightarrow \infty} \bar{M}_{3}, \bar{M}_{3}=\frac{1}{N} \sum_{i=1}^{N} M_{3 i}$,

$$
\begin{gathered}
M_{3 i}=\left(\begin{array}{c}
\tilde{B}_{11 \cdot 2 i}^{-1}\left(B_{12 i} \tilde{B}_{22 i}^{-1}\left(B_{23 i} \tilde{B}_{33 i}^{-1}+\lambda_{2 i}^{\prime}\right)+B_{13 i} \tilde{B}_{33 i}^{-1}\right) \\
\tilde{B}_{22 i}^{-1}\left(B_{23 i} \tilde{B}_{33 i}^{-1}+\lambda_{2 i}^{\prime}\right)+\tilde{B}_{22 i}^{-1} B_{21 i} \tilde{B}_{11 \cdot 2 i}^{-1}\left(B_{12 i} \tilde{B}_{22 i}^{-1}\left(B_{23 i} \tilde{B}_{33 i}^{-1}+\lambda_{2 i}^{\prime}\right)+B_{13 i} \tilde{B}_{33 i}^{-1}\right)
\end{array}\right), \\
M_{4 i}=\left(\begin{array}{cc}
\tilde{B}_{22 i}^{-1} \Sigma_{22 i}^{\frac{1}{2}} & \tilde{B}_{22 i}^{-1}\left(B_{23 i} \tilde{B}_{33 i}^{-1}+\lambda_{2 i}^{\prime}\right) \\
0 & M_{3}
\end{array}\right),
\end{gathered}
$$

$\tilde{\rho}_{i}=\left(B_{21 i}^{\prime}\left(\tilde{B}_{22 i}^{-1}\right)^{\prime} \quad 0\right)$ and

$$
\begin{aligned}
\tilde{\Omega}_{i}^{\frac{1}{2}} & =\left(\begin{array}{cc}
\sigma_{i} \tilde{B}_{11 \cdot 2 i}^{-1} & \tilde{B}_{11 \cdot 2 i}^{-1} M_{1 i} M_{2 i} \\
\sigma_{i} \tilde{B}_{11 \cdot 2 i}^{-1} \tilde{\phi}_{i} & \left(M_{4 i}+\tilde{B}_{11 \cdot 2 i}^{-1} \tilde{\phi}_{i} M_{1 i} M_{2 i}\right)
\end{array}\right) \\
& =\left(\begin{array}{cc}
\tilde{\omega}_{11 i} & \tilde{\Omega}_{12 i} \\
\tilde{\Omega}_{21 i} & \tilde{\Omega}_{22 i}
\end{array}\right) .
\end{aligned}
$$

Furthermore, we have $\tilde{\Omega}_{21 i}=\tilde{\omega}_{11 i} \tilde{\rho}_{i}$ and we define $\tilde{\omega}_{11 \cdot 2 i}=\tilde{\omega}_{11 i}-\tilde{\omega}_{11 i} \tilde{\Omega}_{12 i} \tilde{\Omega}_{22 i}^{-1} \tilde{\rho}_{i}$.

\section{Proof of Lemma 2}

Letting $\varphi_{i}=\tilde{B}_{11 \cdot 2 i}^{-1} B_{12 i}(1) \tilde{B}_{22 i}(1)^{-1}$, we have

$$
\begin{aligned}
T^{-\frac{1}{2}} \bar{Z}_{t} & =\bar{M}_{3} T^{-\frac{1}{2}} \sum_{s=1}^{t} \varepsilon_{3 s}+\frac{1}{N} \sum_{i=1}^{N}\left(\begin{array}{cc}
\tilde{B}_{11 \cdot 2 i}^{-1} & \tilde{\varphi}_{i} \\
\varphi_{i}^{\prime} & \left(\tilde{B}_{22 i}(1)^{-1}+\tilde{B}_{22 i}(1)^{-1} B_{21 i}(1) \varphi_{i}\right)
\end{array}\right) \\
& \times T^{-\frac{1}{2}} \sum_{s=1}^{t}\left(\begin{array}{c}
\varepsilon_{1 \cdot 2 i, s} \\
\varepsilon_{2 i, s}
\end{array}\right)+O_{p}\left(\frac{1}{\sqrt{N T}}\right)+O_{p}\left(\frac{1}{\sqrt{T}}\right),
\end{aligned}
$$

from which it follows that as $N, T \rightarrow \infty$

$$
\begin{aligned}
T^{-\frac{1}{2}} \bar{Z}_{t} & =\bar{M}_{3} T^{-\frac{1}{2}} \sum_{s=1}^{t} \varepsilon_{3 s}+O_{p}\left(\frac{1}{\sqrt{N}}\right)+O_{p}\left(\frac{1}{\sqrt{N T}}\right)+O_{p}\left(\frac{1}{\sqrt{T}}\right) \\
& \stackrel{w}{\longrightarrow} M_{3} W_{3} .
\end{aligned}
$$


This proves (a).

Moreover, by combining (a) with (A5), we find that as $N, T \rightarrow \infty$

$$
T^{-\frac{1}{2}} \tilde{Z}_{i, t}^{+} \stackrel{w}{\longrightarrow} \tilde{\Omega}_{i}^{\frac{1}{2}} W_{i}
$$

which proves (b).

Analogous to the prove of Lemma 1, we have

$$
\begin{aligned}
\sum_{t=2}^{T} Q_{\tilde{W}} \tilde{Z}_{i, t-1}^{+}\left(Q_{\tilde{W}} \tilde{Z}_{i, t-1}^{+}\right)^{\prime} & =\sum_{t=2}^{T} \tilde{Z}_{i, t-1}^{+}\left(\tilde{Z}_{i, t-1}^{+}\right)^{\prime}-\sum_{t=2}^{T} \tilde{Z}_{i, t-1}^{+}\left(\tilde{W}_{i, t}\right)^{\prime}\left(\sum_{t=2}^{T} \tilde{W}_{i, t}\left(\tilde{W}_{i, t}\right)^{\prime}\right)^{-1} \\
& \times \sum_{t=2}^{T} \tilde{W}_{i, t}\left(\tilde{Z}_{i, t-1}^{+}\right)^{\prime} \\
& =\sum_{t=2}^{T} \tilde{Z}_{i, t-1}^{+}\left(\tilde{Z}_{i, t-1}^{+}\right)^{\prime}+O_{p}(T) .
\end{aligned}
$$

Combining this with (b) we obtain as $N, T \rightarrow \infty$

$$
\begin{aligned}
T^{-2} \sum_{t=2}^{T} Q_{\tilde{W}} \tilde{Z}_{i, t-1}^{+}\left(Q_{\tilde{W}_{i, t-1}} \tilde{Z}^{+}\right. & =T^{-2} \sum_{t=2}^{T} \tilde{Z}_{i, t-1}^{+}\left(\tilde{Z}_{i, t-1}^{+}\right)^{\prime}+O_{p}\left(T^{-1}\right) \\
& \stackrel{w}{\longrightarrow} \tilde{\Omega}_{i}^{\frac{1}{2}}\left(\int W_{i} W_{i}^{\prime}\right)\left(\tilde{\Omega}_{i}^{\frac{1}{2}}\right)^{\prime} .
\end{aligned}
$$

This proves (c).

Finally,

$$
\begin{aligned}
\sum_{t=2}^{T} Q_{\tilde{W}} \tilde{Z}_{i, t-1}^{+}\left(Q_{\left.\tilde{W} \tilde{\varepsilon}_{1 \cdot 2 i, t}\right)}\right. & =\sum_{t=2}^{T} \tilde{Z}_{i, t-1}^{+} \tilde{\varepsilon}_{1 \cdot 2 i, t}-\sum_{t=2}^{T} \tilde{Z}_{i, t-1}^{+}\left(\tilde{W}_{i, t}\right)^{\prime}\left(\sum_{t=2}^{T} \tilde{W}_{i, t}\left(\tilde{W}_{i, t}\right)^{\prime}\right)^{-1} \\
& \times \sum_{t=2}^{T} \tilde{W}_{i, t}\left(\tilde{\varepsilon}_{1 \cdot 2 i, t}\right) \\
& =\sum_{t=2}^{T} \tilde{Z}_{i, t-1}^{+} \tilde{\varepsilon}_{1 \cdot 2 i, t}+O_{p}(T) O_{p}\left(T^{-1}\right) O_{p}(\sqrt{T}) .
\end{aligned}
$$

Thus,

$$
\begin{aligned}
T^{-1} \sum_{t=2}^{T} Q_{\tilde{W}} \tilde{Z}_{i, t-1}^{+}\left(Q_{\tilde{W}} \tilde{\varepsilon}_{1 \cdot 2 i, t}\right) & =T^{-1} \sum_{t=2}^{T} \tilde{Z}_{i, t-1}^{+} \tilde{\varepsilon}_{1 \cdot 2 i, t}+O_{p}\left(T^{-\frac{1}{2}}\right) \\
& \stackrel{w}{\longrightarrow} \sigma_{i} \tilde{\Omega}_{i}^{\frac{1}{2}} p_{i}\left(W_{i}\right)
\end{aligned}
$$

as $N, T \rightarrow \infty$. This proves (d) and hence the proof of Lemma 2 is complete.

The proof of Theorem 2 follows similar arguments as the proof of Theorem 3. However, if $k<m+1, \tilde{\Omega}_{i}^{\frac{1}{2}}$ and $\tilde{\Omega}_{22 i}$ are no longer square matrices such that we have to make use of generalized inverse in that case. 
The Wald statistic $w_{\tilde{\delta}_{1 i}}$ is given by

$$
\begin{aligned}
w_{\tilde{\delta}_{1 i}} & =\check{\sigma}_{i}^{-2} \sum_{t=2}^{T} Q_{\tilde{W}^{2} \tilde{\varepsilon}_{1 \cdot 2 i, t}}\left(Q_{\tilde{W}} \tilde{Z}_{i, t}^{+}\right)^{\prime}\left(\sum_{t=2}^{T} Q_{\tilde{W}} \tilde{Z}_{i, t-1}^{+}\left(Q_{\tilde{W}} \tilde{Z}_{i, t-1}^{+}\right)^{\prime}\right)^{-1} \\
& \times \sum_{t=2}^{T} Q_{\tilde{W}} \tilde{Z}_{i, t-1}^{+}\left(Q_{\tilde{W}} \tilde{\varepsilon}_{1 \cdot 2 i, t}\right)
\end{aligned}
$$

where $\tilde{\sigma}_{i}^{2}$ is $\hat{\sigma}_{i}^{2}$ with $Q_{\tilde{W}}$ in place of $Q_{W}$. By using the same steps as for $\hat{\sigma}_{i}^{2}$ in Theorem 2, we obtain $\tilde{\sigma}_{i}^{2} \stackrel{p}{\longrightarrow} \sigma_{i}^{2}$ as $N, T \rightarrow \infty$. This result, together with Lemma 2 (c) and (d), implies that as $N, T \rightarrow \infty$

$$
\begin{aligned}
w_{\tilde{\delta}_{1 i}} & \stackrel{w}{\longrightarrow} \sigma_{i}^{-2} \sigma_{i} \int d W_{1 i} W_{i}^{\prime}\left(\tilde{\Omega}_{i}^{\frac{1}{2}}\right)^{\prime}\left(\tilde{\Omega}_{i}^{\frac{1}{2}} \int W_{i} W_{i}^{\prime}\left(\tilde{\Omega}_{i}^{\frac{1}{2}}\right)^{\prime}\right)^{-1} \sigma_{i} \tilde{\Omega}_{i}^{\frac{1}{2}} \int W_{i} d W_{1 i} \\
& =p_{i}\left(W_{i}\right)^{\prime} P_{i}\left(W_{i}\right)
\end{aligned}
$$

which establishes the required result for (a).

Furthermore, similarly to the prove of Theorem 1, by the rules for partitioned regressions, $T \tilde{\alpha}_{1 i} \stackrel{w}{\longrightarrow} \tilde{d}_{i}$ and $T^{2} \operatorname{var}\left(\tilde{\alpha}_{1 i}\right) \stackrel{w}{\longrightarrow} \tilde{D}_{i}$ as $N, T \rightarrow \infty$, where $\tilde{d}_{i}$ and $\tilde{D}_{i}$ are defined similarly to $d_{i}$ and $D_{i}$ above, but replacing $\omega_{11 i}, \omega_{11 \cdot 2 i}$ and $\Omega_{22 i}$ with $\tilde{\omega}_{11 i}, \tilde{\omega}_{11 \cdot 2 i}$ and $\tilde{\Omega}_{22 i}$ respectively. This yields the required result for (b).

\section{A.3.1 Proof of Corollary 1}

The proof of Corollary 2 is completed by noting that $\tilde{\rho}_{i}=0$ if $X_{i, t}$ is strongly exogenous. 


\section{A.4 Tables}

Table 1: Critical values and moments for the individual $t$-tests.

\begin{tabular}{cccccccc}
\hline \hline & & \multicolumn{3}{c}{ Critical values } & & \multicolumn{2}{c}{ Moments } \\
\cline { 3 - 4 } \cline { 7 - 8 } Model & $m$ & $10 \%$ & $5 \%$ & $1 \%$ & & $\mathrm{E}\left(D_{\tau}\right)$ & $\operatorname{var}\left(D_{\tau}\right)$ \\
\hline 1 & 1 & -2.985 & -3.315 & -3.932 & & -1.709 & 1.069 \\
& 2 & -3.484 & -3.819 & -4.434 & & -2.212 & 1.044 \\
& 3 & -3.883 & -4.219 & -4.848 & & -2.617 & 1.026 \\
& 4 & -4.233 & -4.570 & -5.191 & & -2.965 & 1.020 \\
& 5 & -4.538 & -4.876 & -5.503 & & -3.272 & 1.012 \\
2 & 1 & -3.426 & -3.744 & -4.339 & & -2.250 & 0.884 \\
& 2 & -3.845 & -4.168 & -4.775 & & -2.644 & 0.915 \\
& 3 & -4.199 & -4.528 & -5.138 & & -2.985 & 0.931 \\
& 4 & -4.512 & -4.841 & -5.454 & & -3.287 & 0.943 \\
& 5 & -4.792 & -5.123 & -5.747 & & -3.564 & 0.947 \\
3 & 1 & -3.814 & -4.122 & -4.697 & & -2.704 & 0.779 \\
& 2 & -4.175 & -4.488 & -5.078 & & -3.024 & 0.837 \\
& 3 & -4.494 & -4.815 & -5.411 & & -3.316 & 0.872 \\
& 4 & -4.780 & -5.103 & -5.703 & & -3.589 & 0.892 \\
& 5 & -5.043 & -5.370 & -5.973 & & -3.841 & 0.904 \\
\hline \hline
\end{tabular}

Notes: Model 1 refers to the specification with no deterministic component, while Models 2 and 3 refer to the specifications with an unrestricted constant, and unrestricted constant and trend, respectively. The value $m$ refers to the number of regressors contained in $X_{i, t}$. 
Table 2: Critical values and moments for the individual Wald tests.

\begin{tabular}{|c|c|c|c|c|c|c|}
\hline \multirow[b]{2}{*}{ Model } & \multirow[b]{2}{*}{$m$} & \multicolumn{3}{|c|}{ Critical values } & \multicolumn{2}{|c|}{ Moments } \\
\hline & & $10 \%$ & $5 \%$ & $1 \%$ & $\mathrm{E}\left(D_{w}\right)$ & $\operatorname{var}\left(D_{w}\right)$ \\
\hline \multirow[t]{5}{*}{1} & 1 & 12.209 & 14.291 & 18.726 & 6.979 & 15.188 \\
\hline & 2 & 17.399 & 19.839 & 24.913 & 10.937 & 23.438 \\
\hline & 3 & 22.344 & 25.010 & 30.634 & 14.872 & 31.381 \\
\hline & 4 & 27.108 & 30.061 & 36.132 & 18.785 & 39.317 \\
\hline & 5 & 31.795 & 34.966 & 41.435 & 22.709 & 47.043 \\
\hline \multirow[t]{5}{*}{2} & 1 & 14.821 & 17.081 & 21.870 & 8.944 & 19.467 \\
\hline & 2 & 19.870 & 22.460 & 27.817 & 12.886 & 27.601 \\
\hline & 3 & 24.750 & 27.571 & 33.400 & 16.833 & 35.554 \\
\hline & 4 & 29.484 & 32.542 & 38.789 & 20.756 & 43.392 \\
\hline & 5 & 34.076 & 37.329 & 43.941 & 24.639 & 50.867 \\
\hline \multirow[t]{5}{*}{3} & 1 & 17.525 & 19.940 & 24.973 & 11.091 & 23.396 \\
\hline & 2 & 22.424 & 25.113 & 30.674 & 14.988 & 31.266 \\
\hline & 3 & 27.190 & 30.127 & 36.200 & 18.891 & 39.200 \\
\hline & 4 & 31.840 & 34.992 & 41.404 & 22.767 & 46.941 \\
\hline & 5 & 36.389 & 39.768 & 46.581 & 26.639 & 54.563 \\
\hline \multirow[t]{5}{*}{4} & 1 & 15.769 & 18.012 & 22.789 & 9.964 & 18.782 \\
\hline & 2 & 20.781 & 23.337 & 28.680 & 13.898 & 26.598 \\
\hline & 3 & 25.629 & 28.422 & 34.305 & 17.824 & 34.284 \\
\hline & 4 & 30.368 & 33.430 & 39.625 & 21.756 & 42.014 \\
\hline & 5 & 34.995 & 38.236 & 44.840 & 25.648 & 49.671 \\
\hline \multirow[t]{5}{*}{5} & 1 & 18.412 & 20.800 & 25.830 & 12.093 & 22.321 \\
\hline & 2 & 23.297 & 25.968 & 31.550 & 15.982 & 30.128 \\
\hline & 3 & 28.084 & 31.016 & 37.034 & 19.888 & 37.981 \\
\hline & 4 & 32.708 & 35.839 & 42.256 & 23.757 & 45.521 \\
\hline & 5 & 37.293 & 40.612 & 47.334 & 27.628 & 53.097 \\
\hline
\end{tabular}

Notes: Models 4 and 5 refer to the specifications with a constant, and constant and trend in the error correction term, respectively. See Table 1 for an explanation of the remaining features of the table. 


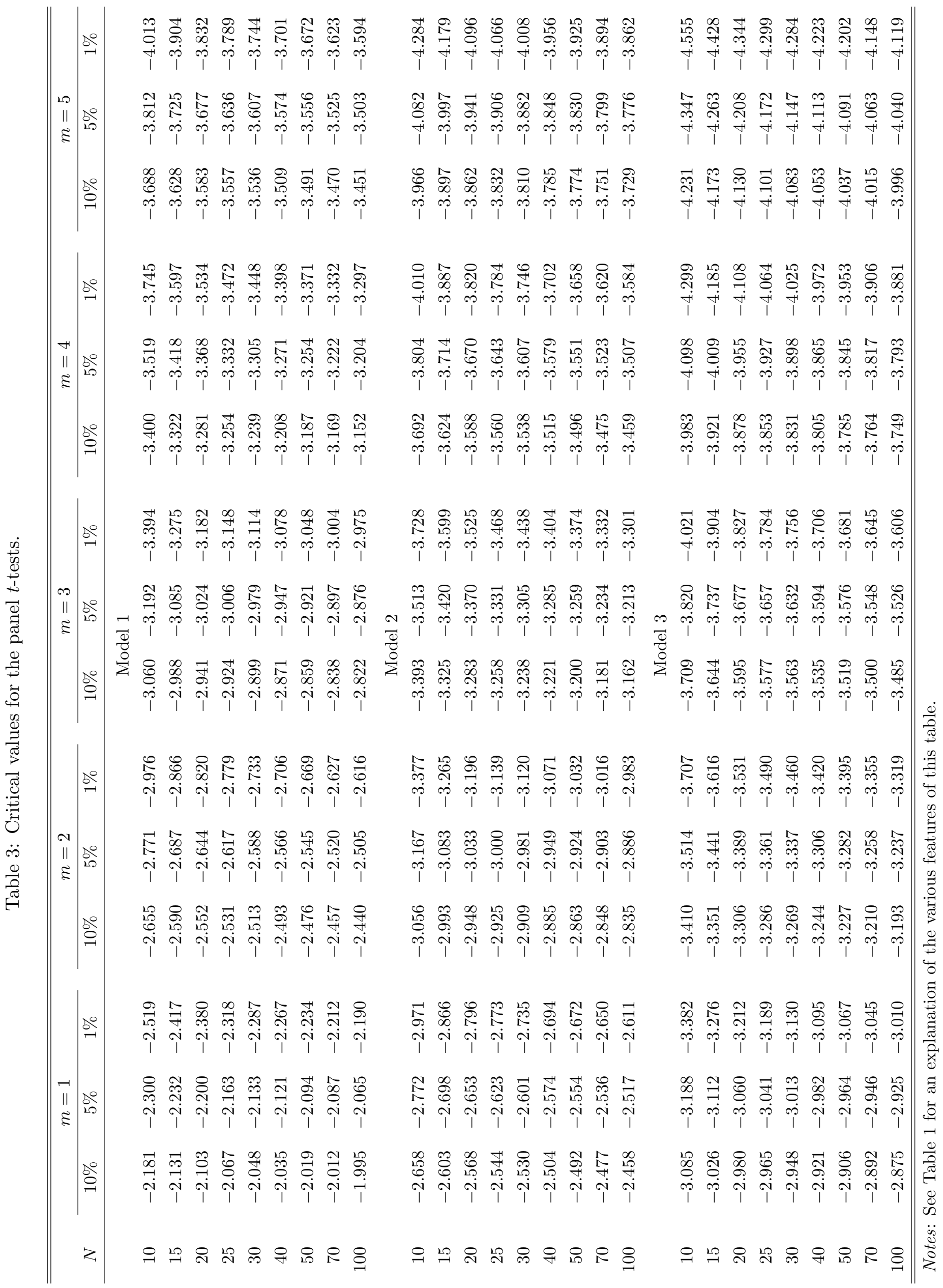




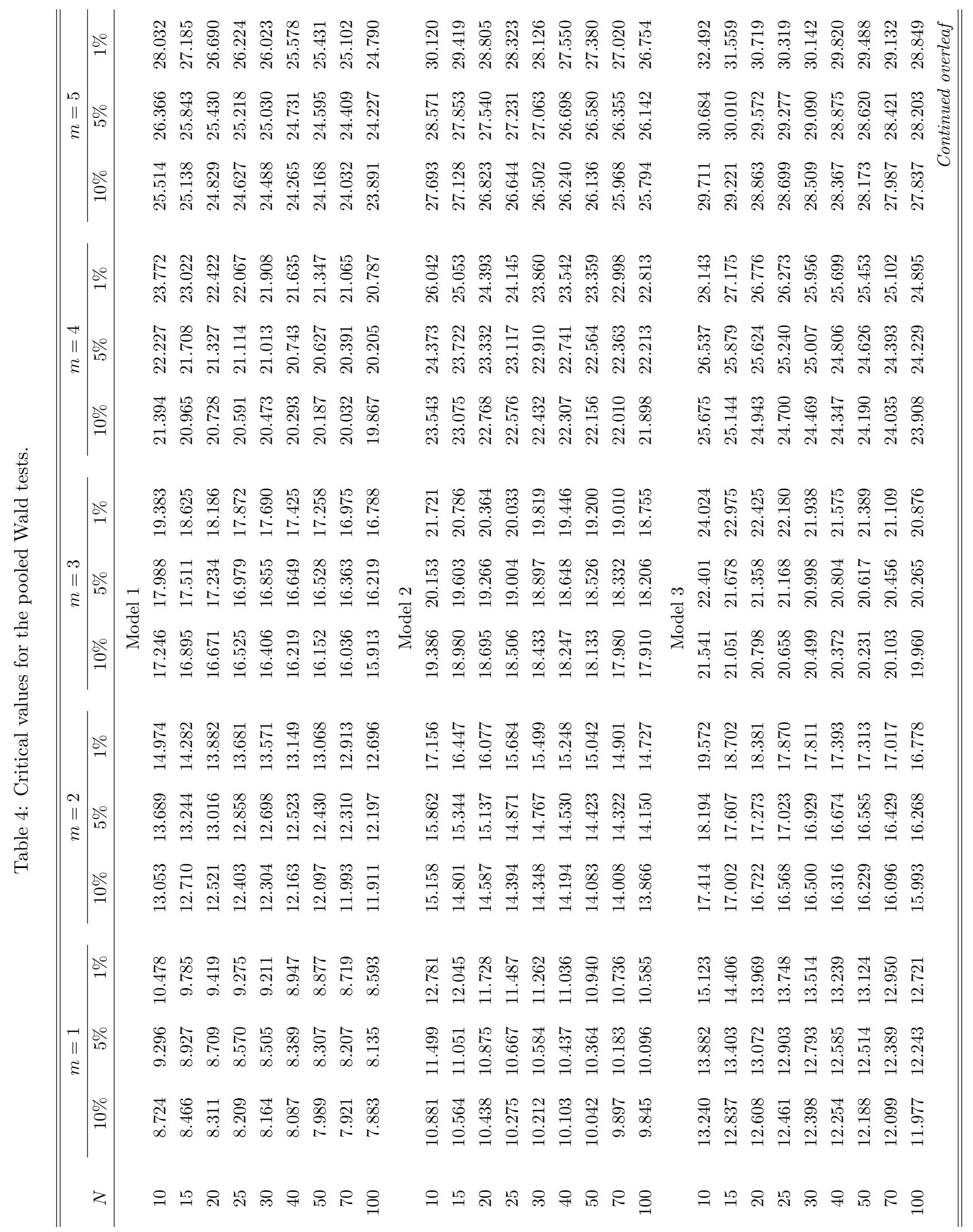




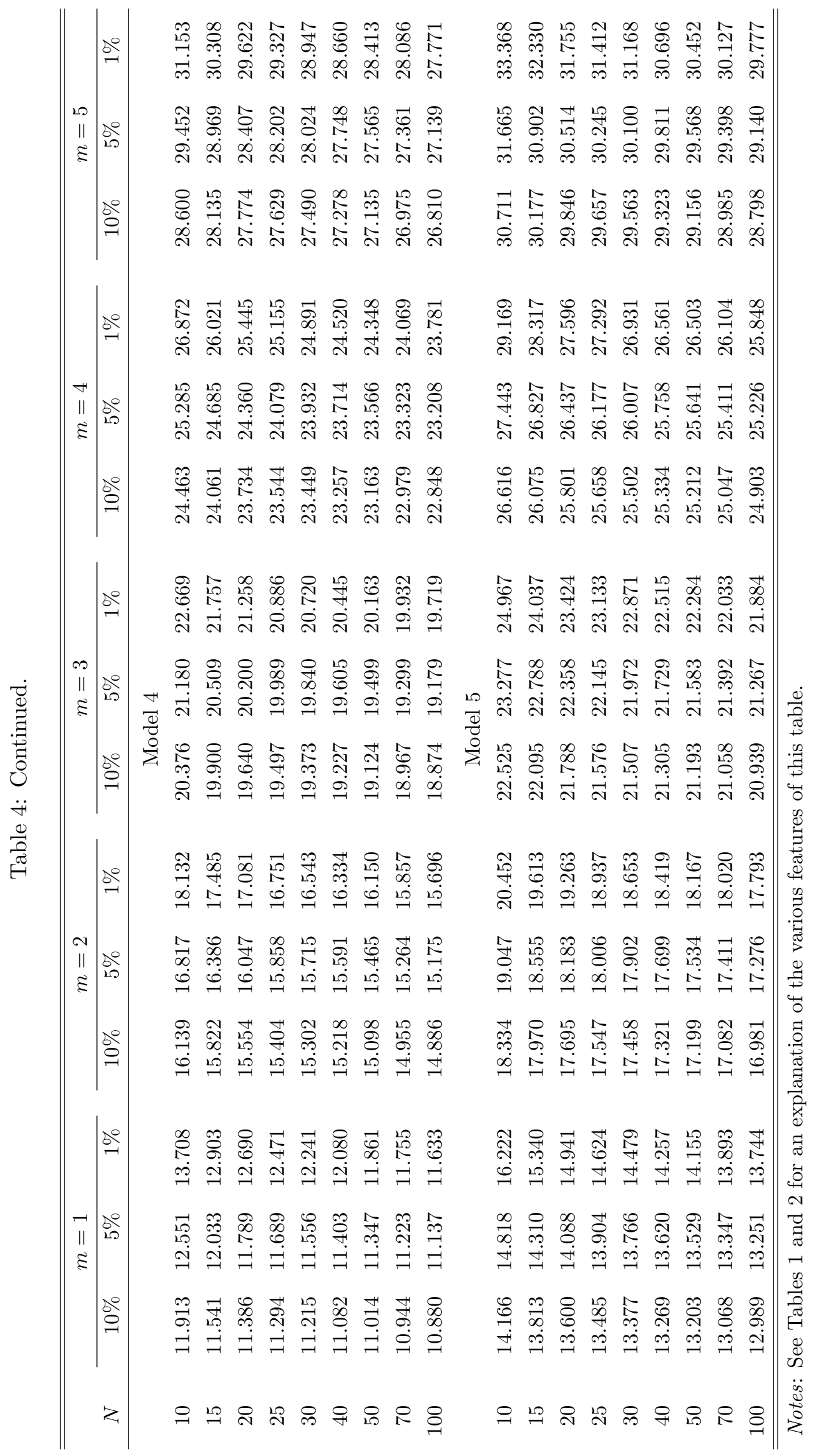




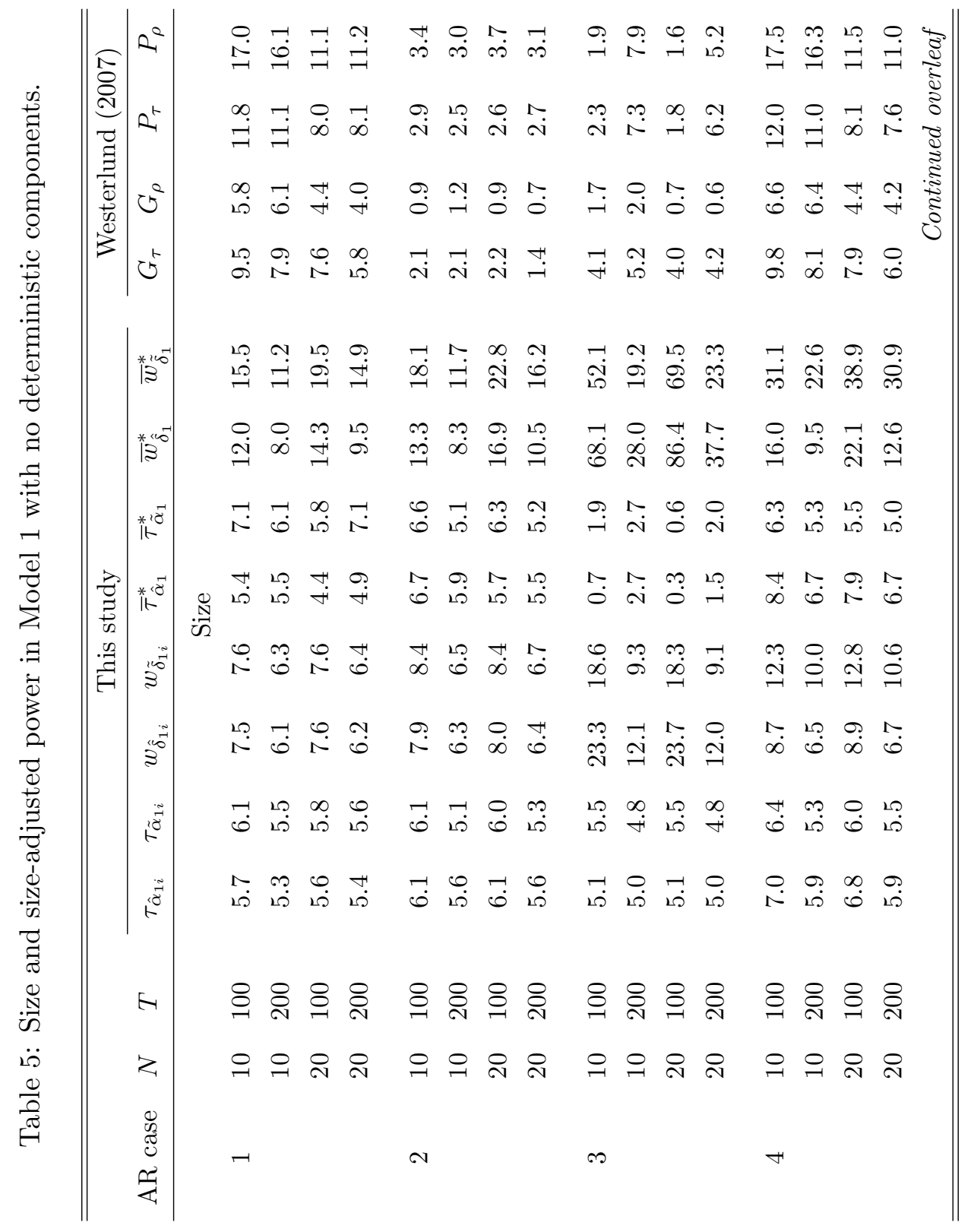




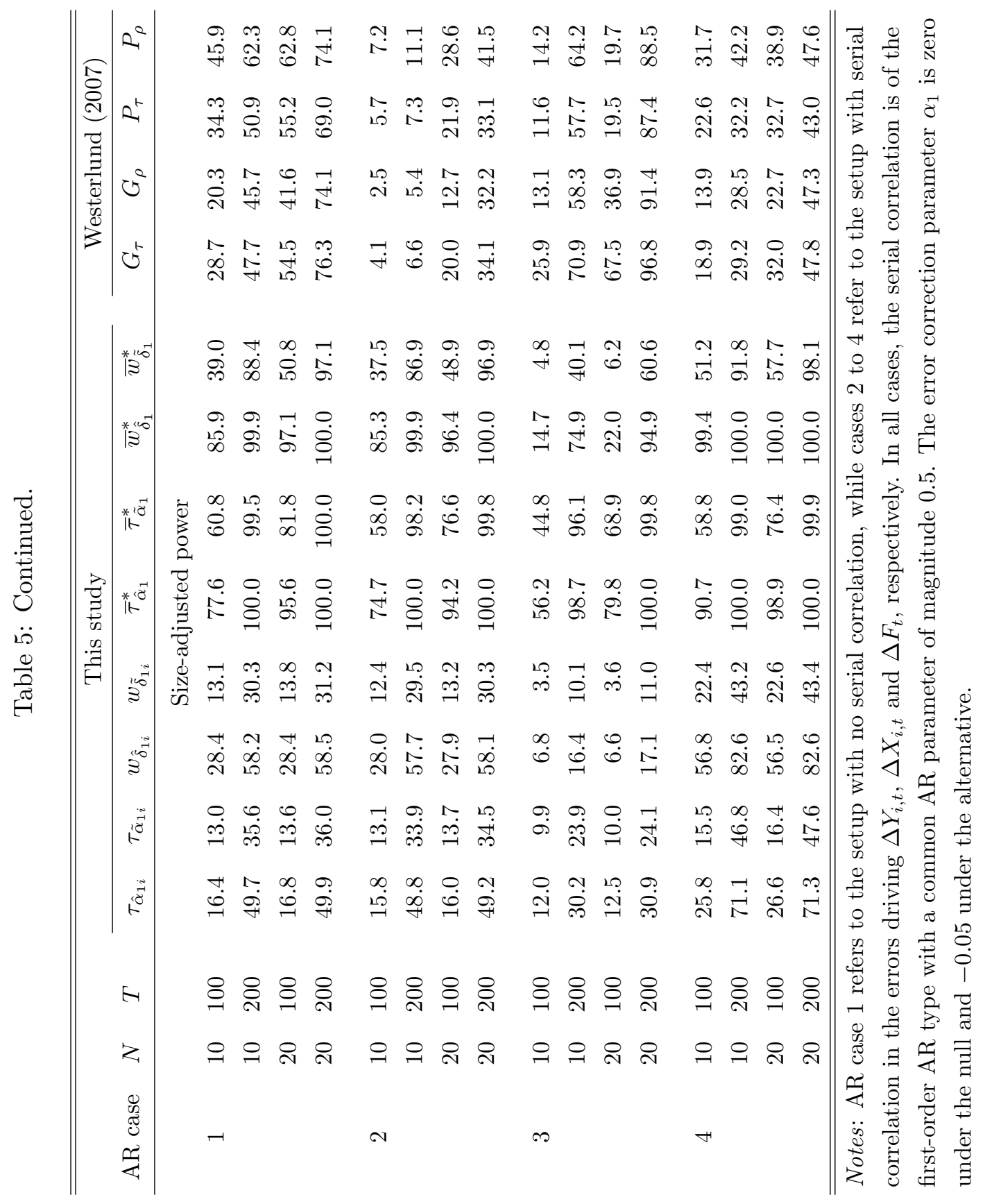




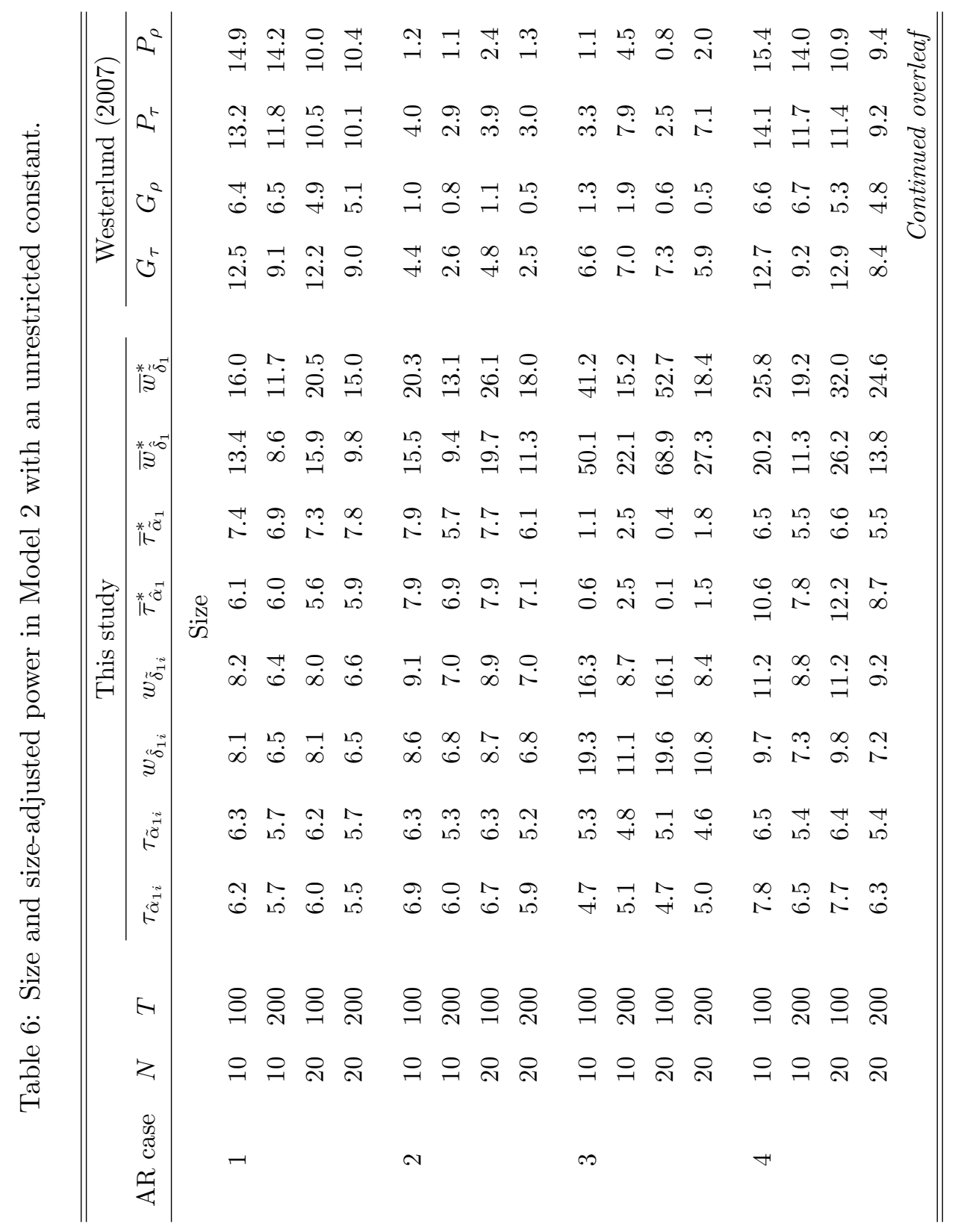




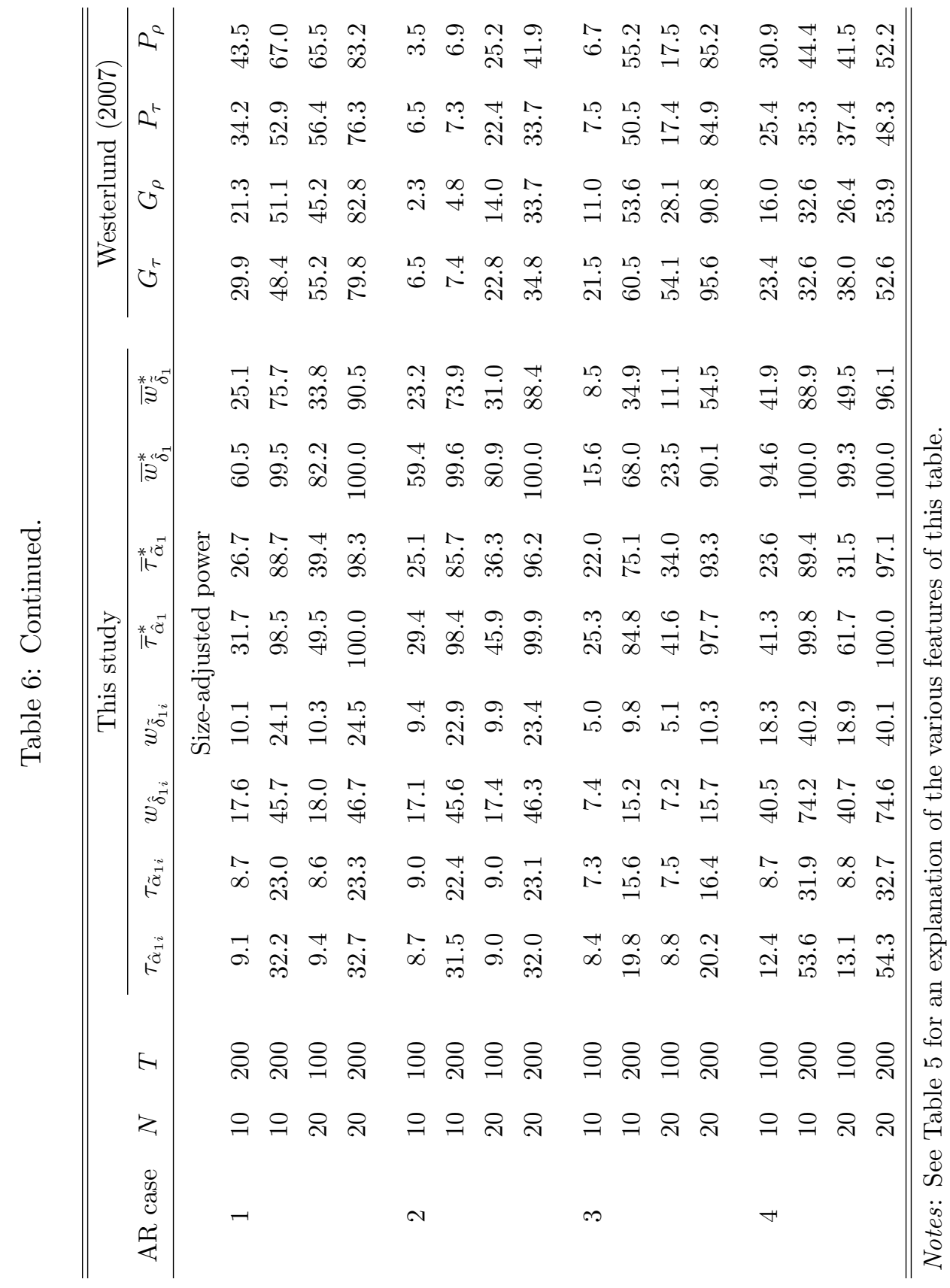




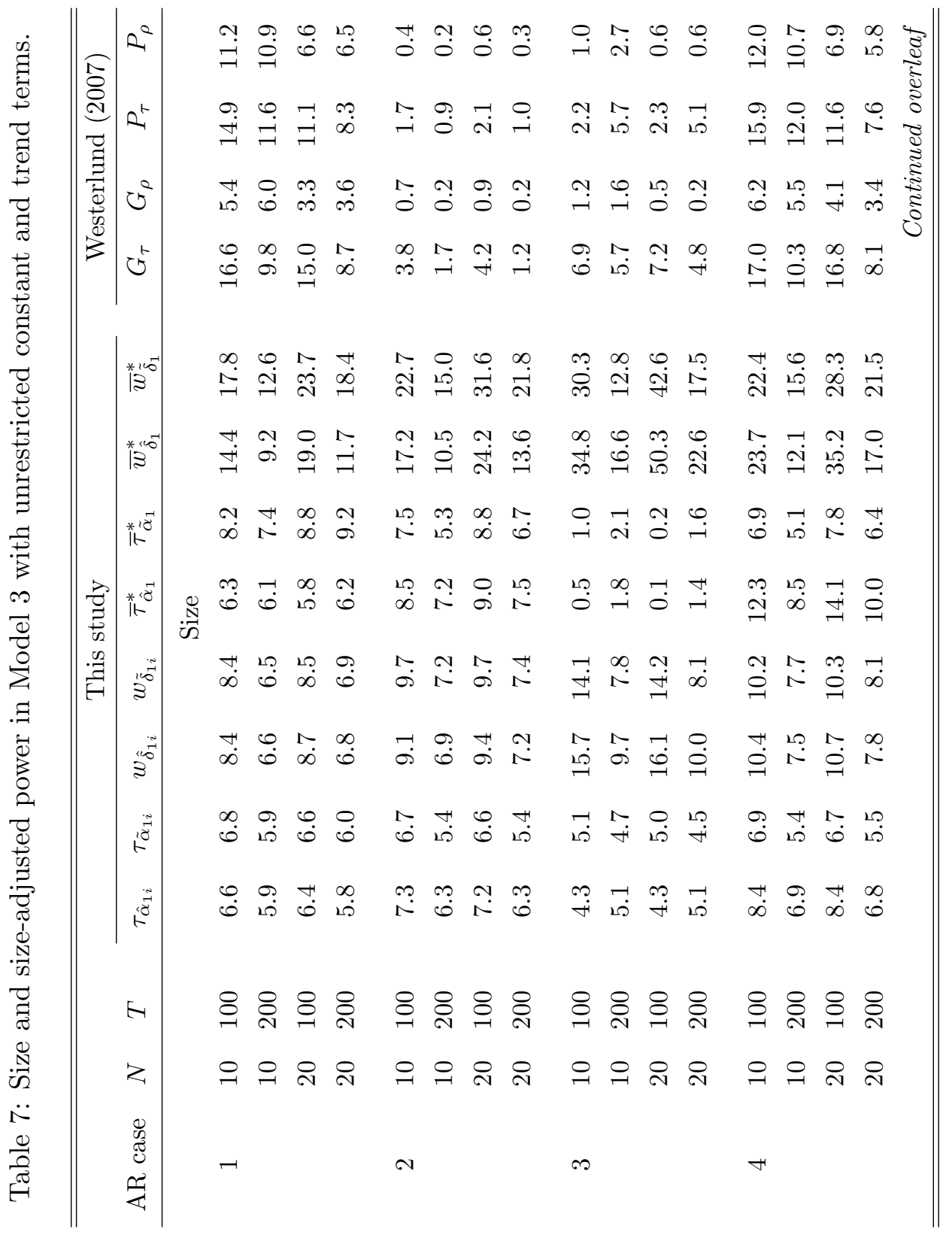




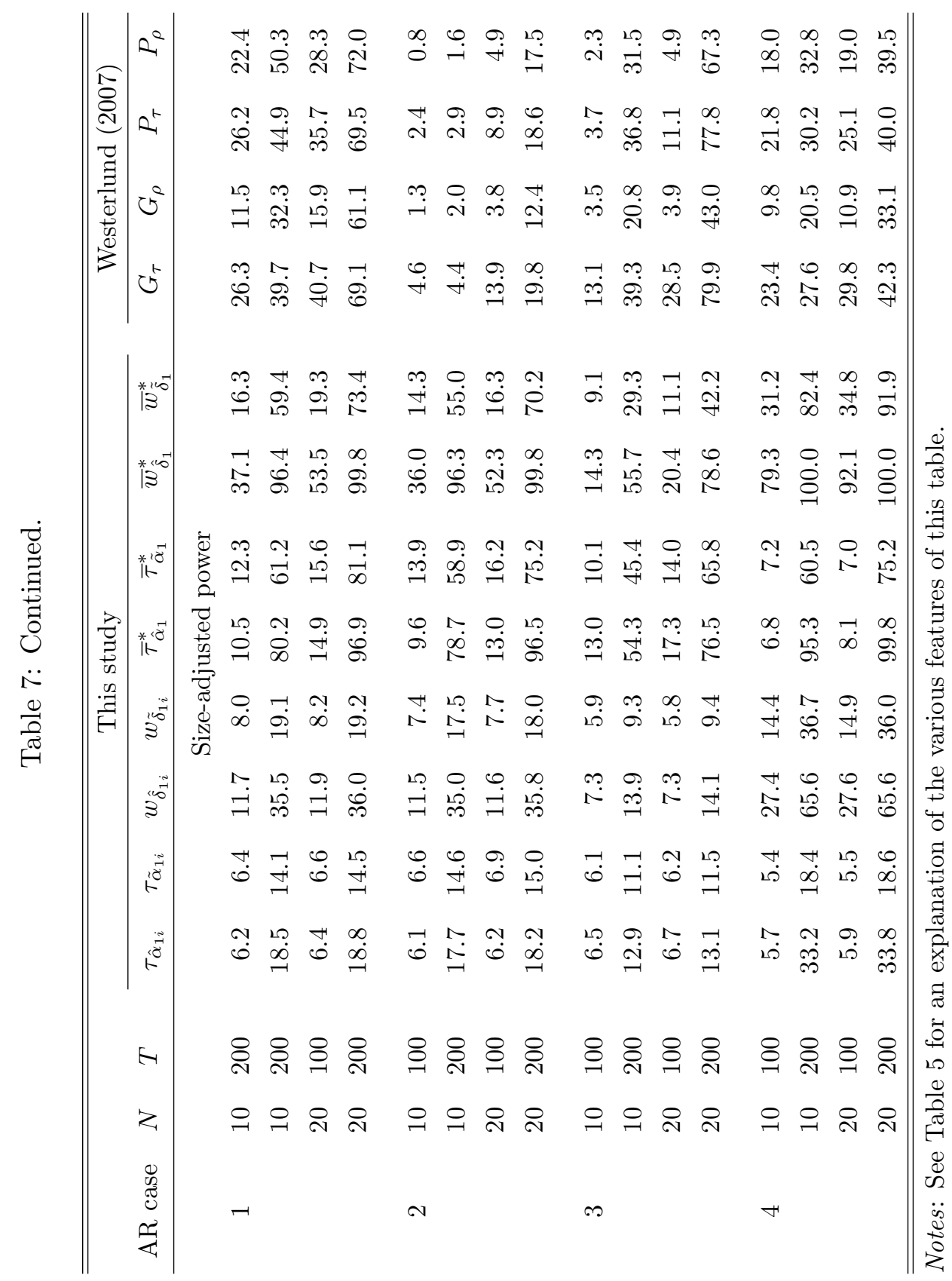




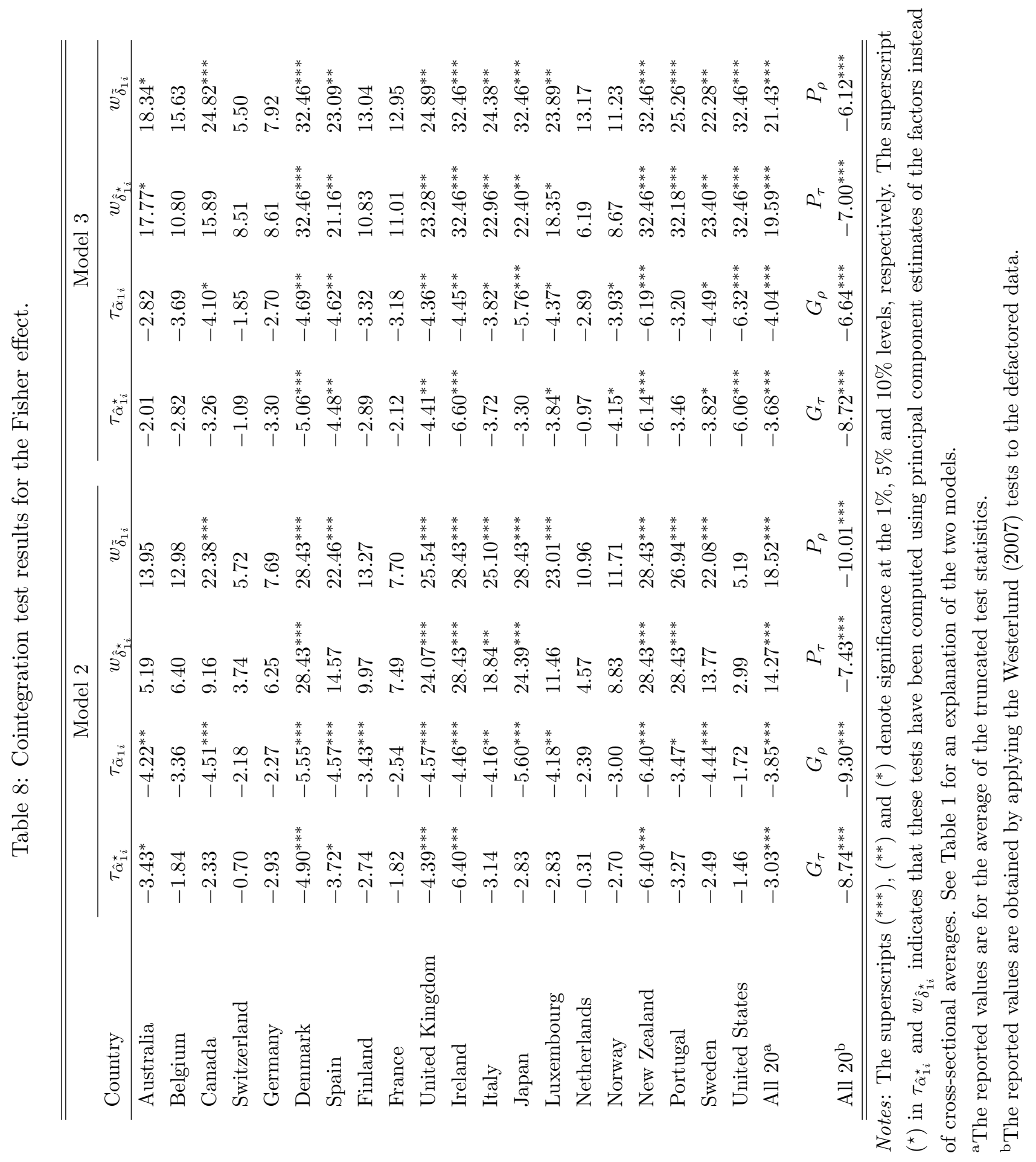




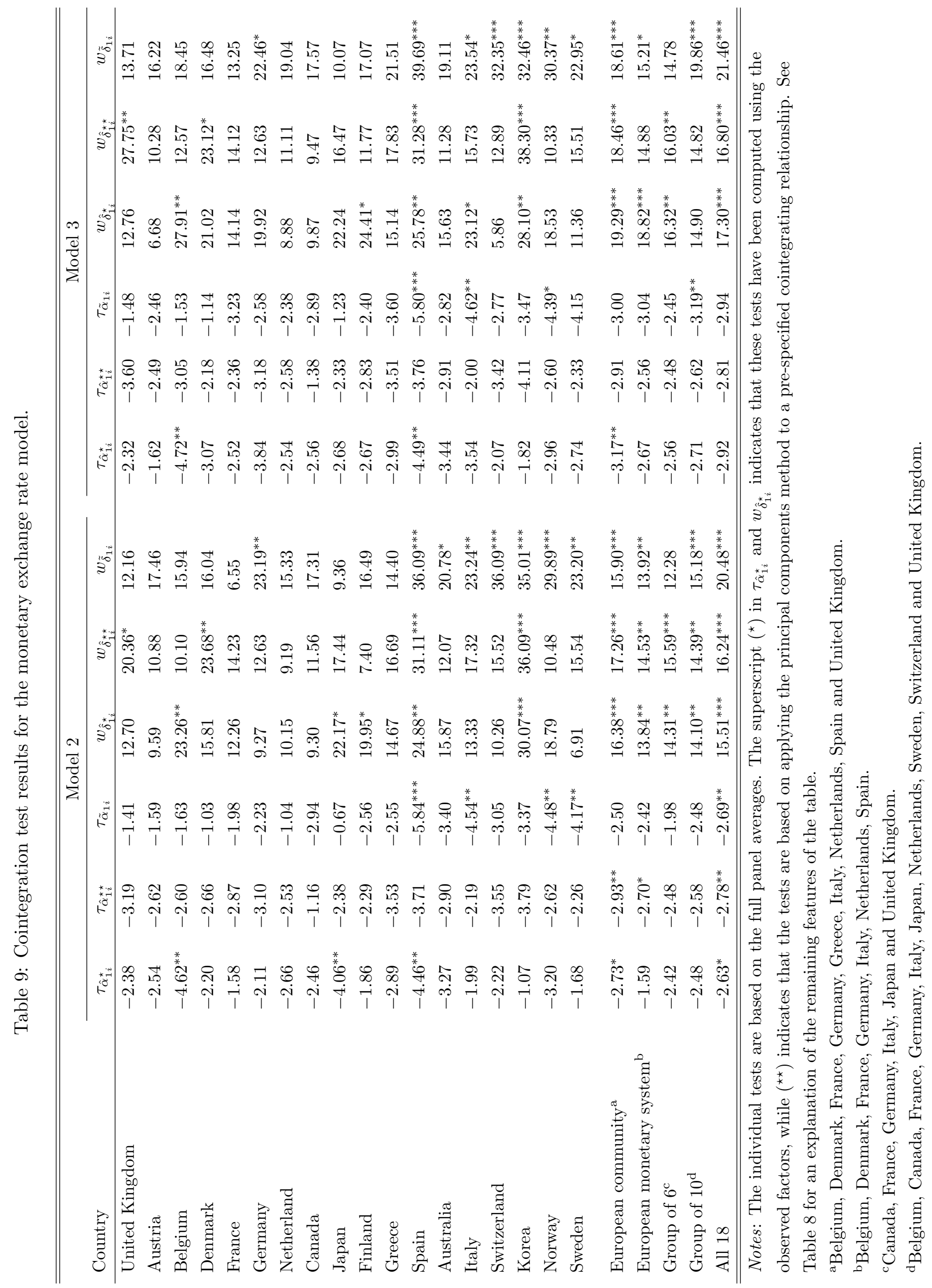


Table 10: Descriptive statistics for the common factors in the monetary exchange rate model.

\begin{tabular}{lcccccc}
\hline \hline & \multicolumn{2}{c}{ Principal components } & & \multicolumn{2}{c}{ Observed } \\
\cline { 2 - 4 } \cline { 6 - 7 } Value & Factor 1 & Factor 2 & Factor 3 & & $m_{t}^{*}$ & $y_{t}^{*}$ \\
\hline AR & 0.97 & 0.98 & 0.97 & & 1.00 & 1.00 \\
SE & 0.03 & 0.05 & 0.02 & & 0.00 & 0.01 \\
ADF & -1.09 & -0.34 & -1.79 & & $-3.06^{* *}$ & -0.42 \\
\hline \hline
\end{tabular}

Notes: AR refers to the estimated first order AR coefficient, SE refers to its standard error and ADF refers to the augmented Dickey and Fuller (1979) test. The autoregressions are fitted with an intercept and the lag orders are determined using the Schwarz Bayesian criterion. See Table 8 for an explanation of the remaining features of the table. 